\title{
Radar observability of near-Earth objects using EISCAT 3D
}

\author{
Daniel Kastinen $^{1,2}$, Torbjørn Tveito ${ }^{3}$, Juha Vierinen ${ }^{3}$, and Mikael Granvik ${ }^{4,5}$ \\ ${ }^{1}$ Swedish Institute of Space Physics (IRF), 98192 Kiruna, Sweden \\ ${ }^{2}$ Department of Physics, Umeå University, 90187 Umeå, Sweden \\ ${ }^{3}$ Department of Physics and Technology, Arctic University of Norway (UiT), 9037 Troms $\varnothing$, Norway \\ ${ }^{4}$ Division of Space Technology, Luleå University of Technology, 98128 Kiruna, Sweden \\ ${ }^{5}$ Department of Physics, University of Helsinki, 00014 Helsinki, Finland
}

Correspondence: Daniel Kastinen (daniel.kastinen@irf.se)

Received: 27 March 2020 - Discussion started: 8 April 2020

Accepted: 15 June 2020 - Published: 15 July 2020

\begin{abstract}
Radar observations can be used to obtain accurate orbital elements for near-Earth objects (NEOs) as a result of the very accurate range and range rate measureables. These observations allow the prediction of NEO orbits further into the future and also provide more information about the properties of the NEO population. This study evaluates the observability of NEOs with the EISCAT 3D $233 \mathrm{MHz}$ $5 \mathrm{MW}$ high-power, large-aperture radar, which is currently under construction. Three different populations are considered, namely NEOs passing by the Earth with a size distribution extrapolated from fireball statistics, catalogued NEOs detected with ground-based optical telescopes and temporarily captured NEOs, i.e. mini-moons. Two types of observation schemes are evaluated, namely the serendipitous discovery of unknown NEOs passing the radar beam and the postdiscovery tracking of NEOs using a priori orbital elements. The results indicate that $60-1200$ objects per year, with diameters $D>0.01 \mathrm{~m}$, can be discovered. Assuming the current NEO discovery rate, approximately 20 objects per year can be tracked post-discovery near the closest approach to Earth. Only a marginally smaller number of tracking opportunities are also possible for the existing EISCAT ultra-high frequency (UHF) system. The mini-moon study, which used a theoretical population model, orbital propagation, and a model for radar scanning, indicates that approximately seven objects per year can be discovered using $8 \%-16 \%$ of the total radar time. If all mini-moons had known orbits, approximately $80-160$ objects per year could be tracked using a priori orbital elements. The results of this study indicate that it is feasible to perform routine NEO post-discovery tracking observations using both the existing EISCAT UHF radar
\end{abstract}

and the upcoming EISCAT 3D radar. Most detectable objects are within 1 lunar distance (LD) of the radar. Such observations would complement the capabilities of the more powerful planetary radars that typically observe objects further away from Earth. It is also plausible that EISCAT 3D could be used as a novel type of an instrument for NEO discovery, assuming that a sufficiently large amount of radar time can be used. This could be achieved, for example by time-sharing with ionospheric and space-debris-observing modes.

\section{Introduction}

All current radar observations of near-Earth objects (NEOs), namely asteroids and comets with perihelion distance $q<$ $1.3 \mathrm{au}$, are conducted post-discovery (Ostro, 1992; Taylor et al., 2019). Radar measurements allow for the determination of significantly more accurate orbital elements (Ostro, 1994). They may also allow construction of a shape model (Ostro et al., 1988; Kaasalainen and Viikinkoski, 2012) and provide information about composition based on polarimetric radar scattering properties (Zellner and Gradie, 1976). In some cases, the absolute rotation state of the object can also be determined by tracking the scintillation pattern of the radar echoes (Busch et al., 2010).

The amount of radar observations of NEOs is limited by resources, i.e. there are significantly more observing opportunities during close approaches than there is radar time available on the Arecibo $(2.36 \mathrm{GHz}, 900 \mathrm{~kW})$ and Goldstone DSS-14 $(8.56 \mathrm{GHz}, 450 \mathrm{~kW})$ radars, which are the two radars that perform most of the tracking of asteroids on a routine 
basis. In order to increase the number of radar measurements of NEOs, it is desirable to extend routine NEO observations to smaller radars, such as the existing EISCAT radars or the upcoming EISCAT 3D radar (233 MHz, $5 \mathrm{MW})$, henceforth abbreviated to E3D, which is to be located in FennoScandinavian Arctic. While these radars may not be capable of observing objects nearly as far away as Arecibo or Goldstone or generating high-quality range-Doppler images, these radars are able to produce high-quality ranging.

Smaller radars can be used for nearly continuous observations, and it is possible that they can even contribute to the discovery of NEOs. Kessler et al. (1980) presented an early attempt at discovering meteoroids outside of the Earth's atmosphere using a space-surveillance radar. However, the observation span was only $8 \mathrm{~h}$, and the results were inconclusive, but 31 objects were identified as possible meteoroids. No follow-up studies were conducted.

As a result of the enhanced survey capability with optical telescopes, the discovery rate of NEOs has greatly increased during the last two decades, from 228 NEOs discovered in 1999 to 2436 discovered in 2019. Recent discoveries include significantly more small objects that have close approach distances within 1 LD compared to discoveries made 20 years ago. It is these objects that are often within the reach of smaller radars. The EISCAT UHF system has in fact already been successfully used to track the asteroid $2012 \mathrm{DA}_{14}$ (J. Vierinen, personal communication, 2013), proving the feasibility of these kinds of observations. One of the range-Doppler observations of $2012 \mathrm{DA}_{14}$, using EISCAT UHF, is shown in Fig. 1. It is expected that NEO observations using E3D will be of a similar nature.

When NEOs make a close approach to Earth, they enter a region where the Earth's gravity dominates. Most of the time, objects will make one single pass and then leave this region again. In some rare cases, the objects are temporarily captured by the Earth-Moon system (Granvik et al., 2012; Fedorets et al., 2017). These events are called temporarily captured fly-bys, if the object makes less than one revolution around the Earth, and temporarily captured orbiters, or minimoons, if the object makes one or more revolutions around the Earth. The existence of a population of transient minimoons in the vicinity of the Earth opens up interesting scientific and technological opportunities, such as allowing a detailed characterisation of the NEO population in a size range that is otherwise hard to study empirically and providing easily accessible targets for space missions (Jedicke et al., 2018; Granvik et al., 2013). However, only two mini-moons have been discovered to date, namely $2006 \mathrm{RH}_{120}$ (Kwiatkowski et al., 2009) and $2020 \mathrm{CD}_{3}$ (Fedorets et al., 2020). Hence, there is very little observational data about these objects and very basic questions about the population still remain unanswered. Because mini-moons, as opposed to generic NEOs, are bound to the Earth-Moon system for a significant period of time, there are more opportunities to track or perhaps even discover them using radar.

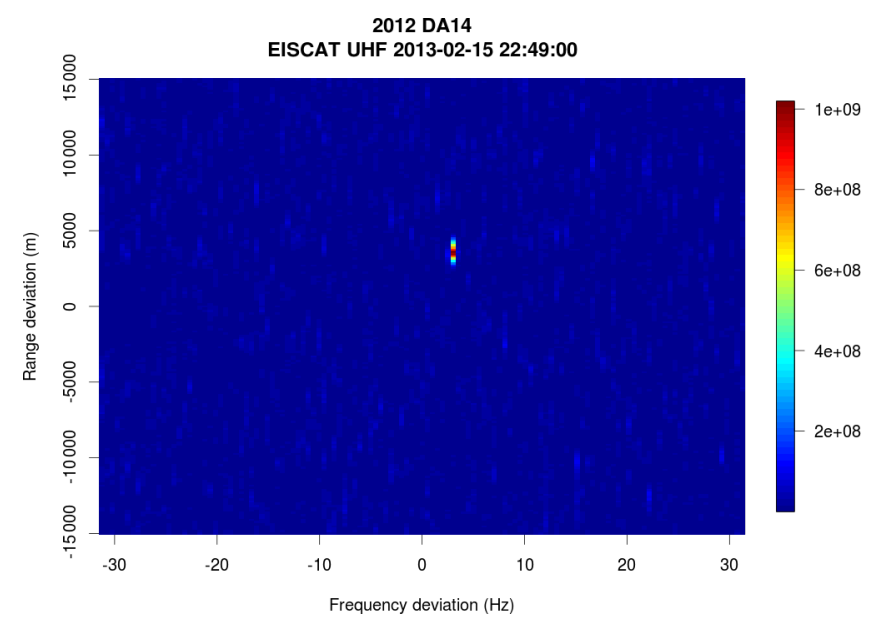

Figure 1. Range-Doppler intensity radar image of asteroid 2012 $\mathrm{DA}_{14}$ observed using EISCAT UHF near the closest approach on 15 February 2013. The Doppler width and range extent are limited due to the low frequency and limited range resolution $(150 \mathrm{~m})$. No range or Doppler width information, apart from object average range and average Doppler, is obtained. NEO observations with E3D are expected to be of a similar nature, with a factor of 4 times less Doppler resolution due to wavelength but a factor of 5 more range resolution due to a higher transmission bandwidth.

The EISCAT radars have already been used for around 20 years to observe the statistical distribution of space debris without prior knowledge of the orbital elements (Markkanen et al., 2009; Vierinen et al., 2019b). Space debris is a collective term for the population of artificially created objects in space, especially those in orbit around the Earth. This population includes old satellites and rocket components and fragments from their disintegration and collisions. There are approximately 16000 catalogued space debris objects, and it is estimated that there are 750000 objects larger than $1 \mathrm{~cm}$ in diameter (Braun, 2018). The capability to detect, track, and catalogue these objects is essential for current and future space operations. One of the most useful methods to measure and catalogue space debris is using radar systems. For example, beam park observations, i.e. a single constant pointing direction with high-power, large-aperture radars, are an important source of information when modelling the space debris population (Krisko, 2014; Flegel et al., 2009; Banka et al., 2000). The current EISCAT system also has a history of providing significant contributions to space debris observations, such as the Chinese antisatellite event (Markkanen et al., 2009; Li et al., 2012), the Iridium-Cosmos collision (Vierinen et al., 2009), and the Indian antisatellite event (Vierinen, 2019). The utility of E3D for space debris discovery and tracking has recently been investigated (Vierinen et al., 2017a, 2019a). The study showed that E3D is a capable instrument for observing the space debris object population due to its phased array antenna system, and its multi-static geometry. The focus of this study is to determine if E3D 
could be similarly used to gain information about the population of NEOs.

The space debris application is very closely related to NEO observations as they both entail the discovery and tracking of a population of hard radar targets. Both populations follow a power law distribution in size, i.e. exponential cumulative growth in number as size decreases. There are, however, several differences. The number density of NEOs close enough to the Earth to be detectable with radar is significantly lower. While observability of NEOs using Arecibo and Goldstone has recently been investigated (Naidu et al., 2016), a similar study has not been done for smaller radars such as E3D. Also, we are not aware of any study of the discovery of NEOs using radar. It is thus desirable to investigate the expected observation capability of this new radar system so that observation programmes that will produce useful data can be prioritised.

E3D is the next-generation international atmosphere and geospace research radar in northern Scandinavia. It is currently under construction and is expected to be operational by the end of 2021. E3D will be the first multi-static, phased array, incoherent scatter radar in the world. It will provide essential data to a wide range of scientific areas within geospace science (McCrea et al., 2015). The primary mission for the E3D radar, which has largely defined the radar design, is atmospheric and ionospheric research. However, the radar is also highly capable of observing meteors entering the Earth's atmosphere (Pellinen-Wannberg et al., 2016), tracking orbital debris (Vierinen et al., 2019a), and even mapping the Moon (e.g. Thompson et al., 2016; Campbell, 2016; Vierinen et al., 2017b; Vierinen and Lehtinen, 2009).

The E3D system will initially consist of three sites, namely in Skibotn in Norway, near Kiruna in Sweden, and near Karesuvanto in Finland. Each of these sites will consist of about $10^{4}$ antennas and act as receivers. The Skibotn site will also act as the transmitter with an initial power of $5 \mathrm{MW}$, later to be upgraded to $10 \mathrm{MW}$. The E3D design allows for novel measurement techniques, such as volumetric imaging, aperture synthesis imaging, multi-static imaging, tracking, and adaptive experiments, together with continuous operations. As two of the main features of the system will be high power and flexibility, it is a prime candidate for conducting routine radar observations of NEOs. The technical specifications E3D are given in Sect. 4.

In this work, we investigate two possible use cases for E3D for observing NEOs, namely the (1) discovery of NEOs and (2) post-discovery tracking of NEOs with known a priori orbital elements. The first case resembles that of space debris observations, where objects randomly crossing the radar beam are detected and, based on their orbital elements, are classified as either orbital space debris or natural objects. The second case is the more conventional radar ranging of NEOs based on a priori information about the orbital elements which yields a more accurate orbit solution. This is described in detail in Sect. 3.
We estimate the detectability using three different approaches, roughly categorised as first order, second order, and third order. The first-order model uses a power law population density based on fireball statistics (Brown et al., 2002). This model makes some major simplifications about the similarities of the cross-sectional collecting areas of the Earth and the E3D radar beam for providing an estimate of the total NEO flux observed by the radar. This method is described in Sect. 5 and the population it uses in Sect. 2.1. In Sect. 6 we describe the second-order model that assesses the number of post-discovery tracking opportunities that are expected in the near future. For this model, we use close approaches predicted for the last 12 months by the Center for NEO Studies (CNEOS) catalogue (NASA JPL, 2020), which is described Sect. 2.2. Lastly, the third-order model is a full-scale propagation and observation simulation of a synthetic mini-moon population which is described in Sect. 7. Although we predict that the vast majority of NEOs are not observable by radars due to size and range issues, one interesting and promising subpopulation of NEOs in terms of detectability is the minimoons. This population is described in Sect. 2.3. The results for each method are also given in the respective model description section. Finally, we discuss and draw conclusions based on our results in Sects. 8 and 9.

\section{Near-Earth object population models}

\subsection{Fireball statistics}

Fireball observation statistics can be used to estimate the influx of small NEOs colliding with the Earth. By making the assumption that the flux of NEOs passing nearby the Earth is the same, it is possible to make a rough estimate of the number of objects that cross the E3D radar beam. A synthesis of NEO fluxes estimated by various authors is given by Brown et al. (2002), who estimate a log-log linear relationship between the cumulative number $N_{\mathrm{FB}}$ of NEOs hitting the Earth at diameter $>D$ to be as follows:

$\log _{10} N_{\mathrm{FB}}=a_{0}-b_{0} \log _{10} D$.

Here $a_{0}=1.568 \pm 0.03$ and $b_{0}=2.70 \pm 0.08$. For example, using this formula, one can estimate that the number of objects colliding with the Earth that are larger than $10 \mathrm{~cm}$ is approximately $1.8 \times 10^{4} \mathrm{yr}^{-1}$.

This population model is convenient as it will allow us to theoretically investigate the number of objects detectable by a radar without resorting to large-scale simulations.

\subsection{Known NEOs with close approaches to the Earth}

The Jet Propulsion Laboratory (JPL) CNEOS maintains a database of NEO close approaches. This database contains objects that have close encounters with the Earth and provides information, such as the date and distance for the closest approaches (Chesley and Chodas, 2002). As the database 
consists of known objects, not modelled populations, there are significantly more past encounters than projected future encounters. Many smaller objects, of which only a small fraction are known, are only discovered when they are very close to the Earth. As a test population we use the data provided for approaches within 0.05 au that occurred during one year from 13 March 2019 to 13 March 2020. This population contains 1215 objects, contrasting with 107 objects in the year from 13 March 2020 to 13 March 2021. The database was accessed on 13 March 2020 when there were a total of 149916 objects in the catalogue.

In addition to the closest approach distance, the database provides an estimate of the diameter of each object estimated from the absolute magnitude. The diameter is used to estimate the signal-to-noise ratio (SNR) obtainable for radar observations and both planned and serendipitous discovery observations near the closest approach.

In Fig. 6 we show the distributions of some of the orbital elements in the NEO population being investigated. The reason for the peculiar shape of eccentricity as a function of the semimajor axis (top-left plot in Fig. 6) is that anything in the bottom right of the plot, outside the region populated, would not cross the Earth's orbit. Similarly, the inclination distribution is biased towards low inclinations, simply due to the fact that these objects are more likely to be near the Earth.

The CNEOS catalogue offers a way to judge what a realistic number of tracking opportunities will be for E3D. Because the orbital elements are not known for most smaller NEOs, the primary source for tracking opportunities is newly discovered objects, which are added to the database near closest approach. Approximately $50 \%$ of the objects are discovered before the closest approach and $50 \%$ afterwards, primarily as the objects are approaching from the direction of the Sun and are not observable in the day-lit hemisphere using telescopic surveys. The number of annual detections has been steadily increasing, and we expect significantly more tracking opportunities within the next few years given the constantly improving sky surveys and the start of new surveys, such as the Rubin Observatory Legacy Survey of Space and Time (LSST; Ivezić et al., 2019).

\subsection{Mini-moon model}

Only two mini-moons have been discovered so far, and we therefore have to rely on theoretical predictions of their orbits and sizes rather than a model that is based on direct observational data. The theoretical models are based on a numerical analysis of the NEO capture probability, estimation of the average capture duration, and the estimated flux of NEOs into the capturable volume of phase space. Whereas Granvik et al. (2012) focused on mini-moons only and estimated the flux based on the debiased NEO model by Bottke et al. (2002), Fedorets et al. (2017) extended the model to encompass both orbiters and fly-bys and tied the model to the updated NEO model by Granvik et al. (2016).
Here we use the newer mini-moon model by Fedorets et al. (2017). The average mini-moon makes 3-4 revolutions around the Earth during a capture which lasts about 9 months. The largest mini-moon captured at any given time is about $1 \mathrm{~m}$ in diameter. The realisation of the model contains 20272 synthetic mini-moon orbits, with absolute Vband magnitude $29.6<H_{\mathrm{V}}<37.1$. The epochs of the synthetic mini-moons are randomly spread across the 19-year Metonic cycle to properly average the changes in the mutual geometry of the Earth, the Moon, and the Sun. Hence, about 1000 mini-moons are captured in any given year during the simulation. The lead time for capturing is typically not more than 2-3 months, so the simulated mini-moon environment is in a steady state for 12 months after the epoch of the chronologically first synthetic mini-moon.

We need to convert absolute magnitudes to diameters to find the radar SNR in subsequent calculations. Using the relationship between the absolute magnitude $H_{\mathrm{V}}$, diameter $D$, and geometric albedo $p_{\mathrm{V}}$, a relation between $H_{\mathrm{V}}$, and $D$ can be derived (e.g. Harris and Harris, 1997; Fowler and Chillemi, 1992) as follows:

$\log _{10}(D)=3.1236-0.5 \log _{10}\left(p_{\mathrm{V}}\right)-0.2 H_{\mathrm{V}}$.

Here it is assumed that the integral bolometric Bond albedo is approximately equal to the Bond albedo in the visual colour range, allowing for the use of the geometric albedo. Here we assume a geometric albedo $p_{\mathrm{V}}=0.14$ (Granvik et al., 2012; Morbidelli et al., 2020), which will transform the modelled population in such a way that the distribution is not only shape representative of the true population but also magnitude representative. In other words, if we simulate $N$ possible object measurements in a certain parameter space region, this corresponds to an expected $N$ real object measurements.

We also need an estimate of the NEO rotation rate because it affects the SNR of a radar measurement (Sect. 3). The rotation-rate distribution of objects smaller than $5 \mathrm{~m}$ in diameter is unfortunately not well constrained. The rotation rate appears to increase exponentially with decreasing size (Bolin et al., 2014; Pravec et al., 2002). Virtually unbiased radar observations have also revealed that very few small NEOs rotate slowly (Taylor et al., 2012; Benner et al., 2015). In what follows, we assume that the objects could have one of four different rotation rates, namely 1000, 5000, 10000 , or 86400 revolutions per day. These values are also consistent with the modelling of cometary meteoroids. For example, Čapek (2014) studied the distribution of rotation rates of meteoroids ejected from 2P/Encke and found that objects with diameters between 1 and $10 \mathrm{~cm}$ have rotation rates approximately between 10 to $0.1 \mathrm{~Hz}$. There are also indirect observations of meteoroid rotation rates derived from optical meteor light curve oscillations. Beech and Brown (2000) estimate $\sim 20 \mathrm{~Hz}$ and less for objects larger than $10 \mathrm{~cm}$ in diameter. 

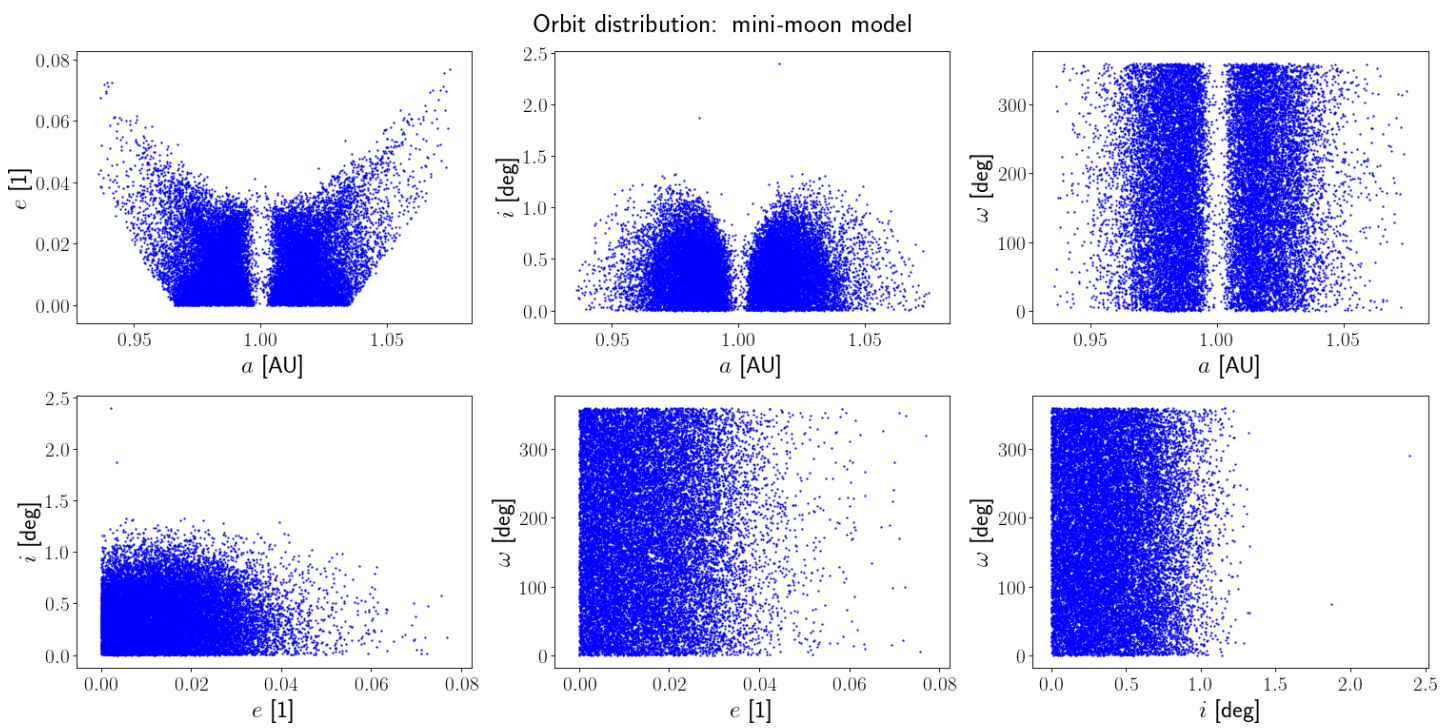

Figure 2. Orbital elements of the synthetic mini-moons in the heliocentric J2000 frame. This is the initial distribution for the mini-moon simulations. We have not illustrated the longitude of the ascending node as this will be closely related to the temporal distribution of objects.

\section{Radar detectability}

When observing NEOs with radar, the most important factor is radar detectability, which depends on the SNR. SNR is determined by the following factors specific to an object, namely diameter, range, Doppler width, and radar albedo, and factors specific to the radar system, namely antenna size, transmit power, wavelength, and receiver system noise temperature. The following model for radar detectability presented here is similar to the one given by Ostro (1992), with slight modifications and additions.

The measured Doppler bandwidth is a combination of relative translation and rotation of the observing frame and the intrinsic rotation of the observed object around its own axis. However, in all cases considered by this study, the effect of a moving observation frame is negligible. As such, the Doppler width $B$ of a rotating rigid object depends on the rotation rate around its own axis and the diameter of the object, as follows:

$B=\frac{4 \pi D}{\lambda \tau_{\mathrm{s}}}$

Here $D$ is the object diameter, $\lambda$ is the radar wavelength, and $\tau_{\mathrm{s}}$ is the rotation period of the object. The Doppler width can be used to determine the effective noise power entering the radar receiver. By coherently integrating the echo $B^{-1} \mathrm{~s}$, we obtain a spectral resolution that corresponds to the Doppler width. When dealing with a pulsed radar, we also need to factor in the transmit duty cycle $\gamma$, which effectively increases the noise bandwidth. The noise power can be written as follows:

$$
P_{\mathrm{N}}=k T_{\mathrm{sys}} B \gamma^{-1}
$$

Here $k$ is the Boltzmann constant and $T_{\text {sys }}$ is the system noise temperature.

The radar echo power originating from a space object, assuming the same antenna is used to transmit and receive, can be obtained using the radar equation as follows:

$P_{\mathrm{S}}=\frac{P_{\mathrm{TX}} G^{2} \lambda^{2} \sigma}{(4 \pi)^{3} R^{4}}$.

Here $P_{\mathrm{TX}}$ is the peak transmit power, $G$ is the antenna directivity, $R$ is the distance between the radar and the object, and $\sigma$ is the radar cross section of the target.

The radar cross section of NEOs can be estimated using the radar cross section of a dielectric sphere, which is either in the Rayleigh or geometric scattering regime (e.g. Balanis, 1999). The transition between the regimes occurs at approximately $0.2 \lambda$. This is similar to the approach taken by the NASA size estimation model for space debris radar cross sections (Liou et al., 2002), which is validated using scalemodel objects in a laboratory. Resonant scattering is not included in the model as the objects are irregular in shape and, on average, the sharp scattering cross section resonances are smeared out. As we are dealing with natural objects made out of less conductive materials than man-made objects, the radar cross section will be scaled down by a factor from that of a perfectly conducting sphere. This factor is a dimensionless quantity called the radar albedo $\widehat{\sigma} \approx\left|\left(\varepsilon_{\mathrm{r}}-1\right) /\left(\varepsilon_{\mathrm{r}}+2\right)\right|^{2}$, with $\varepsilon_{\mathrm{r}}$ being the relative permittivity of the object. In this study, a commonly used value of $\hat{\sigma}=0.1$ is used (Ostro, 1992). The radar cross section model is thus as follows: 
$\sigma= \begin{cases}\hat{\sigma} \frac{9 \pi^{5}}{4 \lambda^{4}} D^{6} & D<\lambda /(\pi \sqrt{3}) \\ \hat{\sigma} \frac{1}{4} \pi D^{2} & D \geq \lambda /(\pi \sqrt{3}) .\end{cases}$

When detecting an object, we are essentially estimating the power of a complex normal random variable measured by the radar receiver. We assume that the system noise power is known to have a far better precision than the power originating from the space object; thus we ignore the uncertainty in determining the noise power in the error budget. It can then be shown that the variance of the power estimate is as follows:

$\operatorname{Var}\left\{\hat{P}_{\mathrm{S}}\right\}=\frac{\left(P_{\mathrm{S}}+P_{\mathrm{N}}\right)^{2}}{K}$,

where $K$ is the number of independent measurements. It is possible to obtain an independent measurement of power every $B^{-1} \mathrm{~s}$, which means that there are $K=\tau_{\mathrm{m}} B$ measurements for an observation period of length $\tau_{\mathrm{m}}$, assuming that $\tau_{\mathrm{m}} \gg B^{-1}$.

To determine if the measurement is statistically significant or not, a criterion can be set on the relative standard error $\delta$. Using the signal power estimator variance from Eq. (7), $\delta$ is defined as follows:

$\delta=\frac{\mathrm{SD}\left\{\hat{P}_{\mathrm{S}}\right\}}{P_{\mathrm{S}}}=\frac{P_{\mathrm{S}}+P_{\mathrm{N}}}{P_{\mathrm{S}} \sqrt{K}}$.

For example, we can use $\delta=0.05$ as the criterion of a statistically significant detection. In this case, the error bars are $5 \%$ in the standard deviation of the received signal power. We can then determine the number of required samples as follows:

$$
K=\frac{\left(P_{\mathrm{S}}+P_{\mathrm{N}}\right)^{2}}{\delta^{2} P_{\mathrm{S}}^{2}} .
$$

We can also determine the minimum required observation time needed to reduce the relative error to $\delta$ as follows:

$\tau_{\delta}=\frac{\left(P_{\mathrm{S}}+P_{\mathrm{N}}\right)^{2}}{\delta^{2} P_{\mathrm{S}}^{2} B}$.

The commonly used SNR reported for planetary radar targets compares the received power to the standard deviation of the averaged noise floor as follows:

$\rho=\frac{P_{\mathrm{S}}}{P_{\mathrm{N}}} \sqrt{\tau_{\mathrm{m}} B}$.

In the case of geometric scatter, this is as follows:

$\rho=\frac{1}{4^{\frac{9}{2}} \pi^{\frac{7}{2}} k} \frac{P_{\mathrm{TX}} \gamma G^{2} \lambda^{\frac{5}{2}}}{T_{\mathrm{sys}}} \frac{\widehat{\sigma} d^{\frac{3}{2}} \tau_{\mathrm{s}}^{\frac{1}{2}}}{R^{4}} \tau_{\mathrm{m}}^{\frac{1}{2}}$,

where the equation is grouped into a constant, a radardependent term, an object-dependent term, and the observation duration.

\subsection{Serendipitous detectability}

The above considerations for the detectability of a space object assume that there is a good prior estimate of the orbital elements, which allows radial trajectory corrections to be made when performing the coherent and incoherent averaging. If the objective is to discover an object without prior knowledge of the orbit, one must perform a large-scale grid search in the radial component of the trajectory space during detection. In this case, it is significantly harder to incoherently average the object for long periods of time while matching the radial component of the trajectory with a matched filter; the search space would simply be too large. For space debris targets, we estimate the longest coherent integration feasible at the moment to be about $\tau_{\mathrm{c}}=0.2 \mathrm{~s}$. This also corresponds to the longest observing interval. We will use this as a benchmark for the serendipitous discovery of NEOs. In this case, we need to evaluate the measurement bandwidth using the following:

$B=\max \left(\frac{4 \pi D}{\lambda \tau_{\mathrm{s}}}, \frac{1}{\tau_{\mathrm{c}}}\right)$,

where the bandwidth has a lower bound, which is determined either by the rotation rate or by the coherent integration length. In most cases, the receiver noise bandwidth will be determined by the coherent integration length $B=\tau_{\mathrm{c}}^{-1}$. We will use this in the subsequent studies of serendipitous detectability.

Assuming that we cannot perform incoherent averaging without a priori knowledge of the orbital elements, the SNR will then be as follows:

$\rho=\frac{P_{\mathrm{S}}}{P_{\mathrm{N}}}$

which does not include any effects of incoherent averaging of power.

\section{EISCAT 3D}

The E3D Stage 1 is expected to be commissioned by the end of 2021. It will then consist of one transmit and receive site in Skibotn, Norway, $\left(69.340^{\circ} \mathrm{N}, 20.313^{\circ} \mathrm{E}\right)$ and two receive sites in Kaiseniemi, Sweden, $\left(68.267^{\circ} \mathrm{N}, 19.448^{\circ} \mathrm{E}\right)$ and Karesuvanto, Finland $\left(68.463^{\circ} \mathrm{N}, 22.458^{\circ} \mathrm{E}\right)$. Each of these sites will consist of a phased array with about $10^{4}$ antennas, which will allow for rapid beam steering.

The transmitter in Skibotn will initially have a peak power of $5 \mathrm{MW}$, later to be upgraded to $10 \mathrm{MW}$. For this study, we have assumed a transmit power of $5 \mathrm{MW}$. The transmit duty cycle of the radar is $\gamma=0.25$ or $25 \%$. It will not be possible to transmit continuously with full peak power in a manner that planetary radars conventionally operate. At the same time, the beam on/off switching time for E3D is only a few microseconds, opposed to $5 \mathrm{~s}$ for DSS-14 and 
Arecibo (Naidu et al., 2016), which makes it possible to observe nearby objects.

The other key radar performance parameters for the Stage 1 build-up of E3D are as follows: peak radar gain of $43 \mathrm{~dB}$ $\left(G_{0}\right)$; receiver noise temperature of $150 \mathrm{~K}$; transmitter bandwidth of $\leq 5 \mathrm{MHz}$; receiver bandwidth of $\leq 30 \mathrm{MHz}$; and operating frequency of $233 \mathrm{MHz}$ (wavelength of $1.29 \mathrm{~m}$ ). The main lobe beam width is approximately $0.9^{\circ}$. The system will be able to point down to at least a $30^{\circ}$ elevation. As the radar uses a planar-phased array, the gain reduces as a function of zenith angle $\theta$ approximately as $G=G_{0} \cos (\theta)$. The radar will also allow two orthogonal polarisations to be received and transmitted, allowing for polarimetric composition studies of NEO radar cross sections.

Using Eq. (12), it is possible to compare the sensitivities of different radar systems, such as the radar-parameterdependent portion, as follows:

$\rho \propto \frac{P_{\mathrm{TX}} \gamma G^{2} \lambda^{5 / 2}}{T_{\mathrm{sys}}}$.

Using parameters given by Naidu et al. (2016), the Arecibo observatory $2.38 \mathrm{GHz}$ planetary radar is approximately a factor of $1.7 \times 10^{4}$ more sensitive than E3D. The Goldstone DSS-14 system, on the other hand, is a factor of 600 more sensitive than E3D, and E3D, in turn, is approximately a factor of 8 more sensitive than the existing EISCAT UHF.

As E3D will have a lower sensitivity and very short transmit beam on/off switching time compared to conventional planetary radars, it may be possible to use it as a search instrument, as it is possible to observe nearby objects, and the beam has a large collecting volume. The ability to use the phased array antenna to point anywhere quickly with a $120^{\circ} \times 360^{\circ}$ field of view (FOV) can also be used to increased the effective collecting volume when the radar is used for performing the serendipitous discovery of NEOs.

A full FOV scan can be performed relatively quickly. The beam broadens as the radar points to lower elevations making the scan pattern non-trivial to calculate. At the zenith the beam width of $\mathrm{E} 3 \mathrm{D}$ is about $0.9^{\circ}$, while at $30^{\circ}$ elevation the beam is roughly $4.3^{\circ}$ wide. The broadening only occurs in the elevation direction, i.e. the main lobe becomes elliptical instead of circular (Vierinen et al., 2019a). Using the known relation between broadening and elevation, one can estimate that approximately 1250 beam directions are needed to scan the entire FOV of E3D. An example scanning pattern is illustrated in Fig. 3. Using an integration time of $0.2 \mathrm{~s}$, the entire sky is scanned every $4-5 \mathrm{~min}$.

\section{Discovery of near-Earth objects}

Most $<1 \mathrm{~m}$ diameter NEOs pass the Earth undetected. In order to estimate the feasibility of discovering these objects using radar, we consider a short coherent integration detection strategy that performs a grid search of radial trajecto-

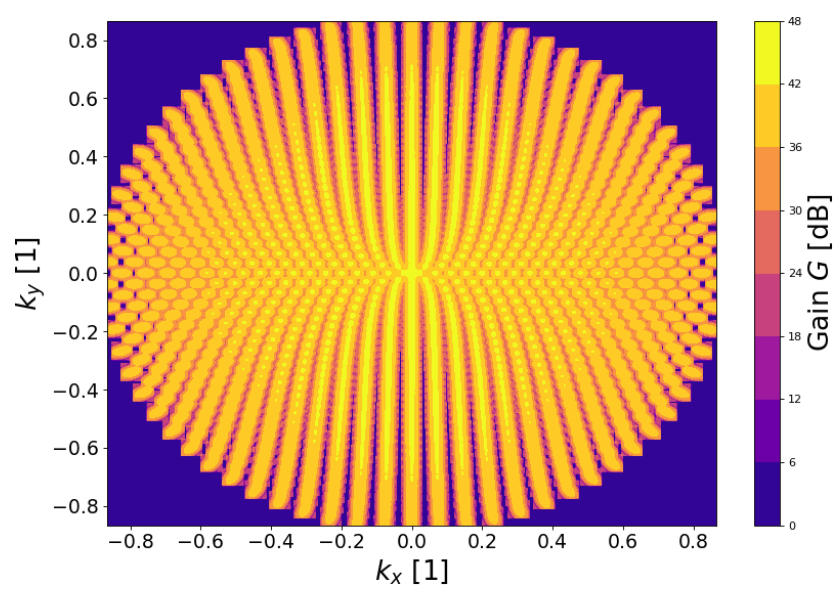

Figure 3. An example full FOV radar scan pattern for E3D including the beam broadening effect. The coordinate axis are the normalised wave vector ground projection $k_{x}, k_{y}$. This scan pattern use approximately $1250 \mathrm{scan}$ direction of $0.2 \mathrm{~s}$ each, completing in about $4-5 \mathrm{~min}$.

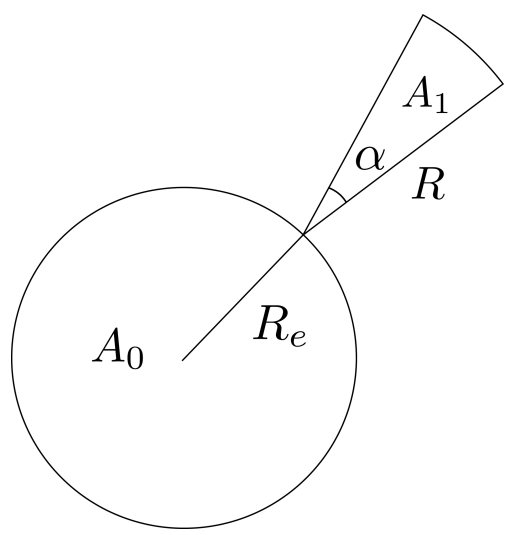

Figure 4. Cross-sectional area of NEOs hitting Earth is indicated by $A_{0}$. The cross-sectional area of NEOs hitting the E3D beam is $A_{1}$, with the radar antenna beam width $\alpha$, and the maximum detectable range $R$.

ries similar to space debris (Markkanen et al., 2009). In the subsequent analysis, a coherent integration length of $0.2 \mathrm{~s}$ is assumed, based on the assumption that integration lengths longer than this would not be computationally feasible. The short coherent integration time also makes it possible to ignore the effect of an object's rotation rate on its detectability, as the effective bandwidth of the coherently integrated signal is nearly always larger than the Doppler bandwidth of the object.

Using the fireball flux reported by Brown et al. (2002), it is possible to estimate the flux of NEOs of various sizes that hit the Earth, as described in Sect. 2.1. We will make the following assumptions: (1) we assume that the flux of objects that pass near Earth is the same as the flux of objects hitting the Earth, (2) we ignore the effects of the Earth's gravity 
on incoming objects, and (3) we assume that all objects approach the Earth aligned with the normal to the meridian circle where E3D is located. It is then possible to treat the Earth and the E3D beam as targets with a certain cross section for incoming NEOs. In this case, the Earth is a circular target with a cross section area $A_{0}=\pi R_{\mathrm{E}}^{2}$, and the E3D radar beam is a target with a cross section area $A_{1}=\frac{1}{2} R(D) \alpha$, where $R(D)$ is the maximum range at which an object of diameter $D$ can be detected. The maximum detectable range $R(D)$ as a function of diameter is shown in Fig. 5. The beam opening angle is $\alpha$, which assumed to be $1^{\circ}$. The cross-sectional areas for Earth-impacting NEOs and NEOs passing the radar beam is depicted in Fig. 4.

The cumulative flux of objects larger than diameter $D$, provided in Eq. (1), can be written as follows:

$N_{\mathrm{FB}}=36.983 D^{-2.7}$.

Differentiating this, we can obtain the density function of objects of diameter $D$ as follows:

$n_{\mathrm{FB}}=99.854 D^{-3.7}$.

The flux density of NEOs crossing the radar beam of a certain size or larger can now be roughly estimated as follows:

$n_{\mathrm{E} 3 \mathrm{D}}(D)=\frac{A_{1}(D)}{A_{0}} n_{\mathrm{FB}}(D)$,

which is in units of objects impacting Earth per year per metre of diameter. When integrated over diameter, we obtain the following cumulative distribution function for the number of radar detections per year of objects with a diameter larger than $D$ :

$N_{\mathrm{E} 3 \mathrm{D}}(D)=\frac{1}{A_{0}} \int_{D}^{\infty} A_{1}\left(D^{\prime}\right) n_{\mathrm{FB}}\left(D^{\prime}\right) \mathrm{d} D^{\prime}$.

The maximum coherent integration time is $\tau_{\mathrm{c}}=0.2 \mathrm{~s}$. However, it takes significantly longer than that for NEOs to drift across the radar beam. Assuming a transverse velocity across the beam of $40 \mathrm{~km} \mathrm{~s}^{-1}$ and detection at a range of $10^{4} \mathrm{~km}$, it takes approximately $\tau=4.4 \mathrm{~s}$ for an object to cross the beam. It is therefore feasible to scan up to $N_{\mathrm{b}}=\tau / \tau_{\mathrm{c}}=22$ different pointing directions with a fencelike scan to increase the collecting area of the radar. In the optimum case, these directions are independent from one another, thus increasing the cumulative number of detections by a factor of $N_{\mathrm{b}}$. While the beam-crossing time is a function of the range, we will use this representative value at $10^{4} \mathrm{~km}$.

The cumulative number of radar detections of objects with $D>1 \mathrm{~cm}$ is estimated to be $\approx 60$ without beam scanning. The lower bound of $1 \mathrm{~cm}$ is determined by the minimum detectable object at the height where ablation becomes significant at $100 \mathrm{~km}$. If a fence-like scan with 20 beam pointing directions is used to increase the total effective collecting area of the radar, the number of detections goes up to
1200 detections per year. The cumulative density function of radar detections per year is shown on the right-hand side of Fig. 5. The blue line indicates radar detections with a fixed radar beam position, and the orange line indicates cumulative detections per year when using a 20-position fence scan. The broken black line indicates the transition from geometric scattering to Rayleigh scattering. Objects smaller than the Rayleigh scattering size limit become much harder to observe due to the vanishing radar cross section, which causes the bend in the cumulative density function.

It is worth noting that the above-mentioned numbers are very rough estimates based on the above simplistic "shotgun" model. However, the results are very promising because the magnitude of the number of serendipitous radar detections of NEOs is an order of magnitude between 10 and 1000, which is significantly larger than 0 . It is therefore plausible that NEOs in the size range $0.01<D<1 \mathrm{~m}$ can be detected using the E3D radar. In order to obtain a more accurate estimate of radar-detection rates, a more sophisticated model of the radar needs to be used together with a realistic NEO population model.

Assuming that objects are in the geometric scattering regime and that the radar antenna aperture is circular, the search-collecting area for a radar is as follows:

$A_{1}(D)=\frac{\pi}{32} \sqrt{\frac{\hat{\sigma}}{k B \rho}} \sqrt{\frac{P \gamma}{T}} \eta d_{r} D$.

Here $B$ is the coherent integration analysis bandwidth, $\rho$ is the minimum SNR required for detection, $d_{r}$ is the diameter of the antenna, and $\eta$ is the aperture efficiency. While a larger antenna is still more advantageous, it is not as crucial for this application as it is for tracking or imaging. The number flux density of NEOs that can be detected when crossing the beam $n_{\mathrm{FB}} A_{1}(D) / A_{0}$ is only linearly dependent on antenna diameter. Factoring everything together, the number flux density of detections per unit diameter is as follows:

$n_{\text {radar }}=\frac{99.854}{32 R_{\mathrm{E}}^{2}} \sqrt{\frac{\hat{\sigma}}{k B \rho}} \sqrt{\frac{P \gamma}{T}} \eta d_{r} D^{-2.7}$.

The cumulative number of radar detections of objects with diameter $>D$ per year, assuming $\hat{\sigma}=0.1, B=5$, and $\rho=$ 10 , is as follows:

$N_{\text {radar }}(D)=5.261 \times 10^{-4} \sqrt{\frac{P \gamma}{T}} \eta d_{r} D^{-1.7}$,

which is valid only when $D>\lambda /(\pi \sqrt{3})$. For example, based on this formula, we can estimate the number of NEOs detected by the Arecibo Observatory $430 \mathrm{MHz}$ ionospheric radar. This system has approximately the following radar performance parameters: $P=10^{6} \mathrm{~W}, d_{r}=305 \mathrm{~m}, \gamma=0.05$, $\eta=0.5$, and $T=100 \mathrm{~K}$. It should be possible to detect approximately 45 NEOs per year with a diameter $>0.15 \mathrm{~m}$ 

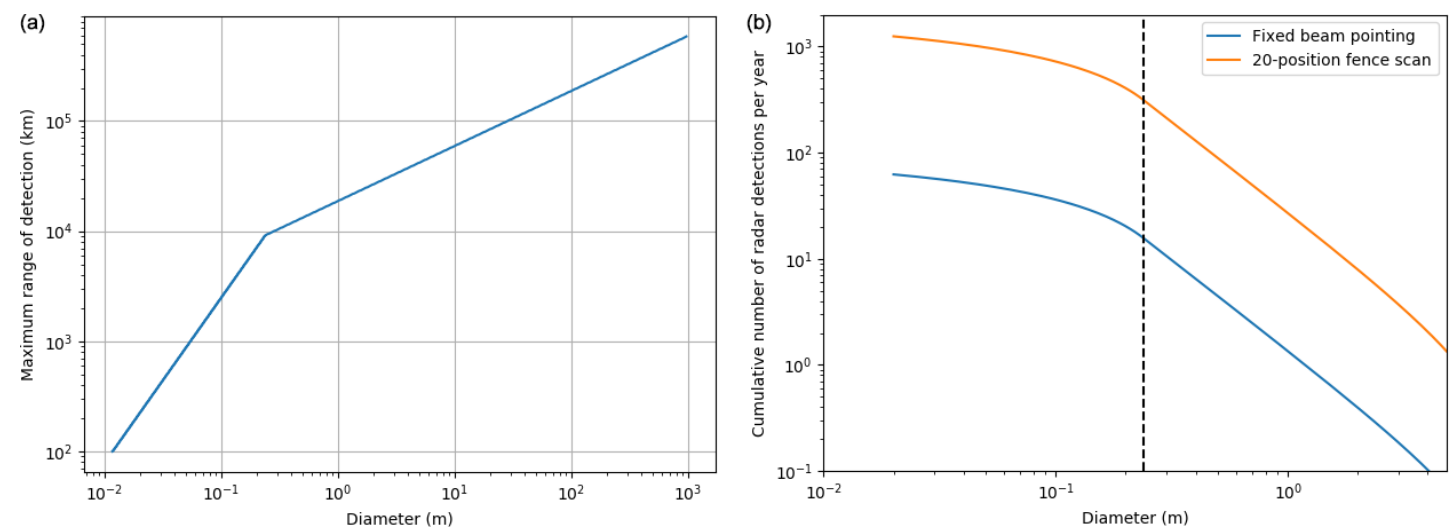

Figure 5. (a) Maximum range at which an object of a certain diameter is detectable with E3D with a $0.2 \mathrm{~s}$ coherent integration length. The range grows initially faster due to object sizes being in the Rayleigh scattering regime, where the radar cross section grows proportionally to $\sigma \propto D^{6}$, and where $D$ is object diameter. Once the transition to geometric scattering occurs, the maximum detectable range grows more slowly. (b) The estimated cumulative number of radar detections per year with E3D, assuming $100 \%$ time use. The blue line is without beam scanning, and the orange line is with a 20-position fence scan. The black broken line indicates the transition from Rayleigh to geometric scattering.

crossing the radar beam, i.e. one detection on average for every $10 \mathrm{~d}$ of continuous operations. Many of these objects should be relatively easy to distinguish from satellites and meteors using Doppler shift and range. While this is a low number of expected detections, it may be feasible to search for these objects as a secondary analysis for ionospheric radar observations.

\section{Observability based on known near-Earth objects}

By applying the methods described in Sect. 3 to the population described in Sect. 2.2, we can determine which objects are observable using the E3D radar facility. Both postdiscovery tracking and serendipitous discovery were investigated. The observability study was performed in two stages. First, we find the SNR for every object only based on range, size, and rotation rate, without considering whether or not the object is in the radar field of view. We only keep objects with an $\mathrm{SNR} \mathrm{h}^{-1}>10$. Then, we use the JPL HORIZONS service to generate an ephemeris for the E3D transmitter location. The ephemeris is used to calculate the maximum SNR when these objects are in the field of view of the radar, keeping only objects with an $\mathrm{SNR} \mathrm{h}^{-1}>10$.

In order to estimate SNR, we require the distance between the radar and the object, the object's diameter, and the rotation rate of the object. The CNEOS database contains the minimum and maximum diameter estimates derived from object absolute magnitude. We use the mean of these two diameter estimates. The HORIZONS ephemeris provides distance and elevation angle during times of observation. Rotation rates are not well known and neither system provides this property for our population of objects.
Bolin et al. (2014) provided two different functions for asteroid rotation period as a function of diameter based on the following two different population samples:

$T_{\mathrm{r}}=0.005 \frac{D}{m}(\mathrm{~h})$,

where $T_{\mathrm{r}}$ denotes the rotation period in hours. This relationship is derived from data of kilometre-sized asteroids. Meteor data suggest a much faster rate of rotation, as follows:

$T_{\mathrm{r}}=0.0001 \frac{D}{m}(\mathrm{~h})$.

These rotation periods differ by a factor of 50 and, as such, influence the number of detectable objects a great deal. Assuming the longer period, we are able to detect 29 out of the 1215 objects. With the shorter estimate, we can only detect 13. We will be using the longer period estimates from now on.

A summary of the characteristics of the objects that can be tracked or detected during the studied 1 year interval is shown in Table 1 . Of the 29 trackable objects, 19 were observable in the last half of 2019. The June to December time period featured two thirds of the observable close approach observation windows. This could possibly be explained through the limited view of the ecliptic plane and small number statistics.

The observable objects were relatively close to the radar, with the shortest range being $0.08 \mathrm{LD}$ and the furthest range being $1.6 \mathrm{LD}$. The diameters of the observable objects ranged between 2.0 and $94 \mathrm{~m}$. The highest $\mathrm{SNR} \mathrm{h}^{-1}$ was $4835,6 \mathrm{had}$ an $\mathrm{SNR} \mathrm{h}^{-1}$ over 1000 , and 12 had an $\mathrm{SNR} \mathrm{h}^{-1}$ over 100. We note that the recently discovered mini-moon, $2020 \mathrm{CD}_{3}$, could have been tracked in April 2019, roughly 10 months before its telescopic discovery. 
Table 1. Objects detectable using E3D during a 1 year interval.

\begin{tabular}{|c|c|c|c|c|c|c|c|c|}
\hline $\begin{array}{l}\text { Object } \\
\text { (ID) }\end{array}$ & $\begin{array}{l}\text { Date } \\
\text { (UTC) }\end{array}$ & $\begin{array}{r}\text { Diameter } \\
(\mathrm{m})\end{array}$ & $\begin{array}{r}\text { Distance } \\
\text { (LD) }\end{array}$ & $\begin{array}{r}\text { Elevation } \\
\left({ }^{\circ}\right)\end{array}$ & $\begin{array}{r}\text { SNR } \\
(\text { per } 0.2 \mathrm{~s}, \mathrm{~dB})\end{array}$ & $\begin{array}{r}\text { SNR } \\
\left(h^{-1}, \mathrm{~dB}\right)\end{array}$ & $\begin{array}{r}\text { Track window } \\
\text { (min) }\end{array}$ & $\begin{array}{r}\text { Detection } \\
\text { window }(\mathrm{min})\end{array}$ \\
\hline $2019 \mathrm{EN}_{2}$ & 14 Mar 2019 03:03 & 13.0 & 0.90 & 65.0 & -15.09 & 11.03 & 1945 & - \\
\hline $2020 \mathrm{CD}_{3}$ & 4 Apr 2019 07:47 & 2.0 & 0.08 & 32.8 & 6.43 & 32.55 & 5195 & - \\
\hline $2019 \mathrm{HE}$ & 20 Apr 2019 23:02 & 19.5 & 0.61 & 37.1 & -8.29 & 17.83 & 2845 & - \\
\hline $2019 \mathrm{JH}_{7}$ & 16 May 2019 00:11 & 5.2 & 0.17 & 61.2 & 5.38 & 31.50 & 1535 & - \\
\hline $2019 \mathrm{KT}$ & 28 May 2019 03:53 & 20.5 & 0.83 & 66.6 & -9.60 & 16.52 & 1665 & - \\
\hline $2019 \mathrm{LZ}_{4}$ & 7 Jun 2019 16:55 & 54.0 & 1.36 & 34.9 & -13.81 & 12.31 & 930 & - \\
\hline $2019 \mathrm{LW}_{4}$ & 8 Jun 2019 15:59 & 15.1 & 0.63 & 80.6 & -6.96 & 19.16 & 2400 & - \\
\hline $2019 \mathrm{MB}_{4}$ & 9 Jul 2019 04:10 & 27.5 & 0.83 & 42.3 & -9.84 & 16.28 & 2090 & - \\
\hline 2019 OK & 25 Jul 2019 07:52 & 94.0 & 1.49 & 30.1 & -11.78 & 14.34 & 840 & - \\
\hline $2019 \mathrm{OD}_{3}$ & 28 Jul 2019 03:16 & 18.0 & 0.48 & 56.3 & -2.00 & 24.12 & 1480 & - \\
\hline $2019 \mathrm{ON}_{3}$ & 29 Jul 2019 02:09 & 11.7 & 0.55 & 49.5 & -9.11 & 17.01 & 335 & - \\
\hline 2019 RQ & 2 Sep 2019 16:50 & 3.2 & 0.28 & 48.9 & -8.12 & 18.01 & 880 & - \\
\hline $2019 \mathrm{SU}_{2}$ & 21 Sep 2019 03:08 & 4.1 & 0.18 & 67.4 & 3.43 & 29.55 & 795 & - \\
\hline $2019 \mathrm{SS}_{2}$ & 21 Sep 2019 06:27 & 3.1 & 0.29 & 48.4 & -9.65 & 16.48 & 425 & - \\
\hline $2019 \mathrm{SS}_{3}$ & 22 Sep 2019 23:48 & 24.5 & 0.73 & 49.6 & -7.46 & 18.66 & 1575 & - \\
\hline 2019 TD & 29 Sep 2019 18:59 & 6.2 & 0.33 & 32.5 & -8.58 & 17.54 & 1200 & - \\
\hline $2019 \mathrm{SM}_{8}$ & 1 Oct 2019 13:56 & 6.2 & 0.40 & 81.8 & -6.56 & 19.56 & 1085 & - \\
\hline $2019 \mathrm{UG}_{11}$ & 1 Nov 2019 21:02 & 19.0 & 0.54 & 42.4 & -5.33 & 20.79 & 1425 & - \\
\hline $2019 \mathrm{VA}$ & 2 Nov 2019 16:23 & 8.8 & 0.28 & 35.2 & -2.05 & 24.07 & 195 & - \\
\hline 2019 VD & 4 Nov 2019 10:56 & 14.4 & 0.46 & 37.0 & -5.97 & 20.15 & 210 & - \\
\hline $2019 \mathrm{UM}_{12}$ & 8 Nov 2019 17:01 & 49.5 & 1.26 & 50.6 & -10.75 & 15.38 & 1315 & - \\
\hline $2019 \mathrm{VF}_{5}$ & 9 Nov 2019 23:01 & 13.7 & 0.49 & 33.2 & -8.31 & 17.81 & 155 & - \\
\hline $2019 \mathrm{WG}_{2}$ & 23 Nov 2019 07:24 & 45.0 & 0.49 & 44.9 & 4.01 & 30.13 & 2420 & - \\
\hline 2019 YB & 17 Dec 2019 23:37 & 5.0 & 0.43 & 60.9 & -10.94 & 15.18 & 1355 & - \\
\hline 2019 YS & 18 Dec 2019 15:27 & 2.1 & 0.16 & 70.5 & 0.20 & 26.32 & 1615 & - \\
\hline $2019 \mathrm{YU}_{2}$ & 23 Dec 2019 19:53 & 14.4 & 0.25 & 54.7 & 7.15 & 33.27 & 1210 & - \\
\hline $2020 \mathrm{BH}_{6}$ & 25 Jan 2020 04:51 & 8.6 & 0.17 & 70.7 & 10.72 & 36.84 & 1925 & 50 \\
\hline $2020 \mathrm{CQ}_{1}$ & 4 Feb 2020 11:46 & 7.3 & 0.16 & 34.4 & 5.81 & 31.93 & 1155 & - \\
\hline $2020 \mathrm{DR}_{4}$ & 25 Feb 2020 22:10 & 4.9 & 0.26 & 30.8 & -6.84 & 19.29 & 170 & - \\
\hline
\end{tabular}

All observable NEOs were above the $30^{\circ}$ cut-off elevation for significantly longer than their maximum incoherent integration time estimate. The minimum observation window was $155 \mathrm{~min}$ and the maximum was over $5000 \mathrm{~min}$. This means that we can expect any serendipitously discoverable objects to be observable for much longer than the time it takes to scan the full field of view, as discussed in Sect. 4. For example, $2020 \mathrm{BH}_{6}$ would have been discoverable for 50 min near its closest approach.

Only a fraction of all objects are discovered and are entered into the CNEOS database. We can assume that there are significantly more objects that could be large enough and have approaches close enough so that E3D would discover them with an all-sky scan. It should be noted that $2012 \mathrm{DA}_{14}$, during its 2013 pass, could also have been easily discoverable with E3D.

Although we have a very limited sample of the total NEO population, it appears that our measurements are not biased towards measuring a specific subset of NEOs with close Earth approaches (Fig. 6). Any potential biases might be revealed once $\mathrm{E} 3 \mathrm{D}$ is in operation, and we can obtain a larger sample space of observable objects.
In order to determine the feasibility of tracking NEO close approaches using the existing EISCAT facilities, we made a similar search for objects observable using the EISCAT UHF radar, which has an antenna gain of $48 \mathrm{~dB}$, transmit power of $P_{\mathrm{TX}}=1.8 \mathrm{MW}$, system noise of $90 \mathrm{~K}$, and duty cycle of $12.5 \%$. The total number of observable objects was 17 , which was a subset of the objects that could be observed using E3D.

The results indicate that it would be feasible to perform routine NEO post-discovery tracking observations using both the upcoming E3D radar and the existing EISCAT UHF radar. This observing programme would nicely complement the capabilities of existing planetary radars, which cannot observe targets that are nearby, due to the long transmit/receiver switching time. Of the $\approx 2000$ NEOs discovered each year, we estimate that approximately $0.5 \%-1 \%$, or approximately 15, can be tracked with E3D or EISCAT UHF when factoring in that around $50 \%$ of the NEOs are discovered before closest approach. 

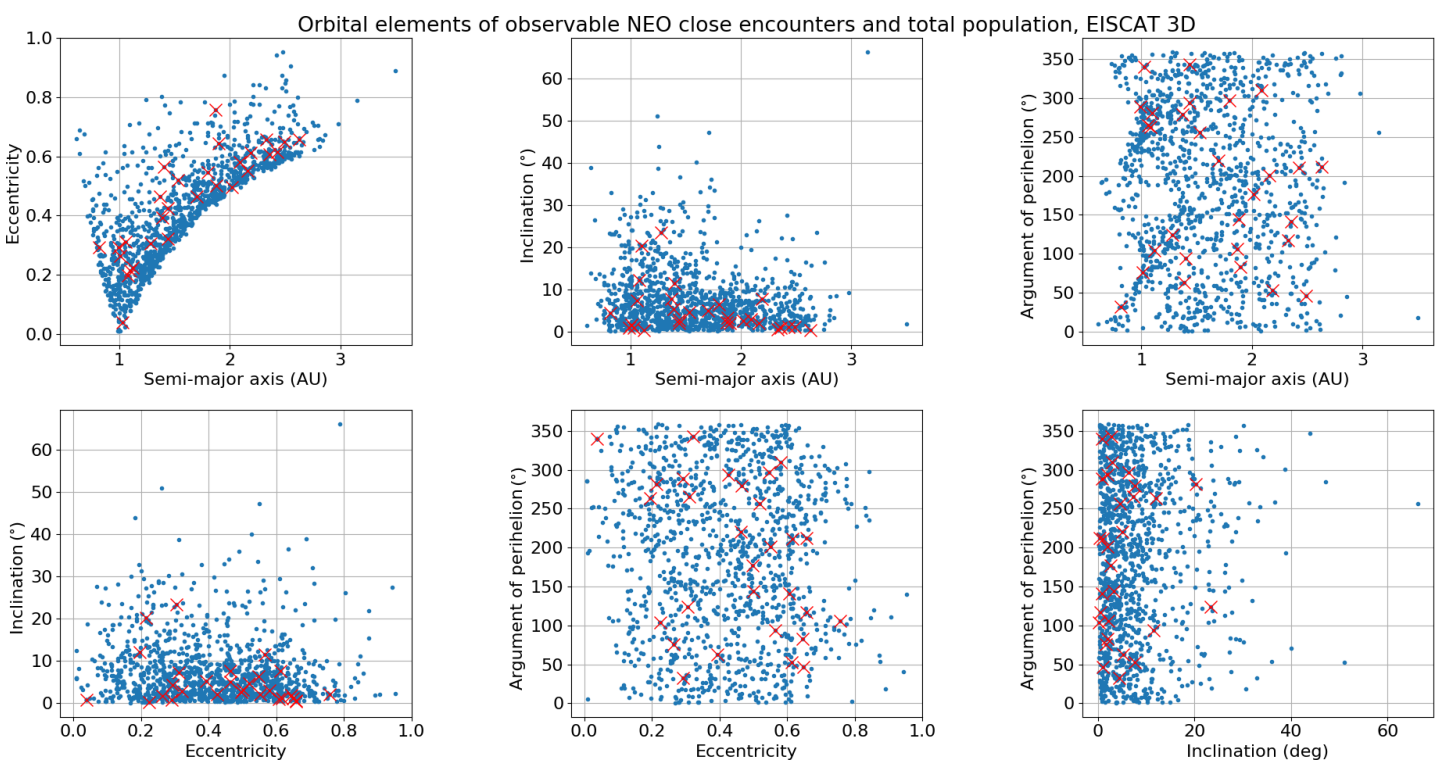

Figure 6. Orbital elements of CNEOS database for the period 13 March 2019 to 13 March 2020, with objects having close approaches to the Earth and being simultaneously observable from the E3D facility, marked with red crosses.

\section{Observability of mini-moons}

To accurately determine the observability of a population, one needs to construct a chain that considers the following:

1. Model of the measurement system (E3D)

2. Model of the population (mini-moons)

3. Temporal propagation of the population (solar system dynamics)

4. The observation itself (a detection window and SNR).

A recent effort to determine the capability of E3D with regards to space debris measurement and cataloguing produced a simulation software called SORTS (Kastinen et al., 2019). SORTS already includes a model of E3D (item 1) and simulated observations of space debris (item 4) suitable for this study. All the parameters given in Sect. 4 are included in the model of E3D used in the simulation, including realistic antenna gain patterns. SORTS also provides the framework for creating the chain between the items (1-4) mentioned previously. Space-debris observations are slightly different from mini-moon and NEO observations due to their size, material, and orbits. To account for this difference, we modified the observation simulation (item 4) according to Sect. 3. Specifically, new SNR formulas from Eqs. (12) and (14) were implemented for use in the NEO and mini-moon simulations. The population model previously described in Sect. 2.3 was translated into the format used by SORTS to allow for the observability simulation (item 2).

SORTS propagates each object of a given population and searches for time intervals where the object is within the FOV of E3D. We consider the effective FOV of E3D to be a $120^{\circ}$ cone centred at the zenith, i.e. we allow for pointing down to a $30^{\circ}$ elevation. We do not need to consider any time delays in pointing as interferometric antenna arrays can close to instantaneously point the radar beams electronically.

The only remaining component of the simulation is an interface with a suitable propagation software (item 3 ). SORTS already includes propagation software but only for objects in stable Earth orbit, i.e. objects that do not transition to hyperbolic orbits in the Earth-centred inertial frame. We have thus chosen to use the Python implementation of the REBOUND propagator $^{1}$ (Rein and Liu, 2012), using the IAS15 integrator (Rein and Spiegel, 2015). This N-body integrator can handle arbitrary configurations of interacting particles and test particles. An interface between the REBOUND propagator and SORTS was implemented, allowing for the use of this propagator in all future simulations. For our application, we only need to propagate for tens of years. As such, we have omitted any radiation-related dynamical effects, such as radiation pressure and Poynting-Robertson drag, as these act on much longer timescales. The integration included all planets and the Moon initialised with the JPL DE430 planetary ephemerides.

The integration was configured to use a time step of $60 \mathrm{~s}$. This step size allows for decent resolution when searching for viable observation windows by the radar system. The initial state for REBOUND was inputted in the J2000 heliocentric ecliptic inertial frame; thus the output was also given in this frame. A standard routine was used to transform to the

\footnotetext{
${ }^{1}$ The REBOUND code is freely available at http://github.com/ hannorein/rebound, last access: 26 July 2019
} 
Earth-centred, Earth-fixed J2000 mean Equator and equinox frame. In this frame the E3D system is fixed in space and observation windows are readily calculated.

\subsection{Results}

All 20265 synthetic mini-moons were integrated for 10 years past their initial epochs. As previously mentioned, we chose to assume that the objects could have one of four different rotation rates, namely $1000,5000,10000$, or 86400 revolutions per day. As such, four different SNRs were calculated for each point in time. It was also assumed that signal integration could not last longer than $1 \mathrm{~h}$, i.e. $\tau_{\mathrm{m}}$ was set to $1 \mathrm{~h}$ or the observation window length if this was shorter than $1 \mathrm{~h}$. For each measurement window, only those with at least one measurement point above $10 \mathrm{~dB}$ SNR at some rotation rate were saved. A fifth SNR was also calculated based on serendipitous discovery, i.e. with a much shorter coherent integration time of $0.2 \mathrm{~s}$. For each of these measurement windows, time series of the object position, velocity, and all five SNRs were saved.

If we assume that we have a prior orbit for these objects, the detections are in essence follow-up tracking measurements, and we can consider the tracking SNRs for observability. The a priori orbit does not have to be of good quality; it need only be sufficiently accurate to restrict the search region in the sky. For these tracking measurements, a total of 1999 out of the 20265 objects (9.9\%) had at least one possible measurement window, assuming the 1000 revolutions per day rotation rate. This number dropped to $7.9 \%, 7.2 \%$, and $5.2 \%$ for 5000,10000 , or 86400 revolutions per day respectively.

Without a prior orbit, we have to consider the SNR for serendipitous discovery. Only a total of 116 objects had an observation with $10 \mathrm{~dB}$ SNR or more. The rotation rate does not affect this SNR in this case, as the noise bandwidth is determined by the coherent integration time. We assume that the coherent integration time is limited to $0.2 \mathrm{~s}$ due to the computational feasibility of performing a massive grid search for all possible radial trajectories that matches the trajectory of the target. This results in an effective noise bandwidth of $B=20 \mathrm{~Hz}$, when factoring in the $25 \%$ duty cycle. This is nearly always higher than the intrinsic Doppler bandwidth of the target.

The distribution of sizes, ranges, and SNRs of observable objects are illustrated in Fig. 8. The sharp cut-off in observable mini-moon passes is due to the minimum SNR conditions that were imposed. For a single object, according to Eq. (12), the parameter dependant on dynamical integration is range. Thus, for an object with a number of possible observation windows, it is primarily the minimum range of these windows that determines their observability and thereby the lack of observability above a certain range. This equation also contains a transition from Rayleigh to geometric scattering as the diameter increases. This transition is illustrated by the "kink" in the cut-off at approximately $0.24 \mathrm{~m}$ in diameter. A promising feature is that even though the majority of detections are made at close range, the lower range of diameters has not been reached by the objects in the model. This indicates the possible existence of smaller objects than those included in the mini-moon model that are observable by E3D. This is supported by the results from Sect. 5 that use a population model with smaller sizes. There is no apparent dynamical coupling with object size and observation window closest range. This is expected as the orbital dynamics are not a function of size in the simulation or in the mini-moon model.

The expected annual detection rate is illustrated in Fig. 9. The distribution is fairly uniform, as expected, and the number of observation windows can thus be averaged over the total time span of the model. Assuming a rotation rate of 1000 revolutions per day, the mean expected detection rate is approximately 162 measurement windows per year. The length distribution of these windows with SNR above $10 \mathrm{~dB}$ is illustrated in Fig. 11. Generally, the observation window is between 1 and $10 \mathrm{~h}$.

The initial orbital element distribution of the observable objects is illustrated in Fig. 7. This illustration should be compared to the initial distribution in Fig. 2. The comparison shows no significant observation biases. However, a dedicated bias study is required for population modelling purposes. It is important to note that the orbits in Fig. 7 are not the detected orbit distribution, as the objects are severely perturbed from Keplerian orbits upon Earth capture.

In Fig. 10 we illustrate the zenith distance for the peak SNR observation point of every observation window. As most capturable objects have low-inclination orbits, most observations are centred around the ecliptic, i.e. at lowelevation angles for a high-latitude radar.

Summary statistics of the observability study can be found in Table 2. The number of observable objects in Table 2 is representative of the expected total number of real minimoons that can be tracked by E3D in future if prior orbital elements are known. Each object can have more than one possible observation window and, on average, each object has approximately 1.5 tracking opportunities. As the number of discovered NEOs is steadily increasing every year with better instrumentation and analysis techniques, a larger fraction of the population will be discovered, allowing these follow-up tracking measurements to be performed.

The number of discoverable objects in Table 2 indicates how many serendipitous mini-moon detections can be made if a full FOV scan, using an integration time of $0.2 \mathrm{~s}$, is continuously performed with E3D. This assumes that the objects passes through one of the scanning beams at least once. The number of discoverable objects gives an average of 6.74 mini-moons discovered per year. As there are only 12 more observation windows than unique objects that are discoverable, a sparse scanning strategy that counts on observing one of many possible passes of the same object is not possible. 

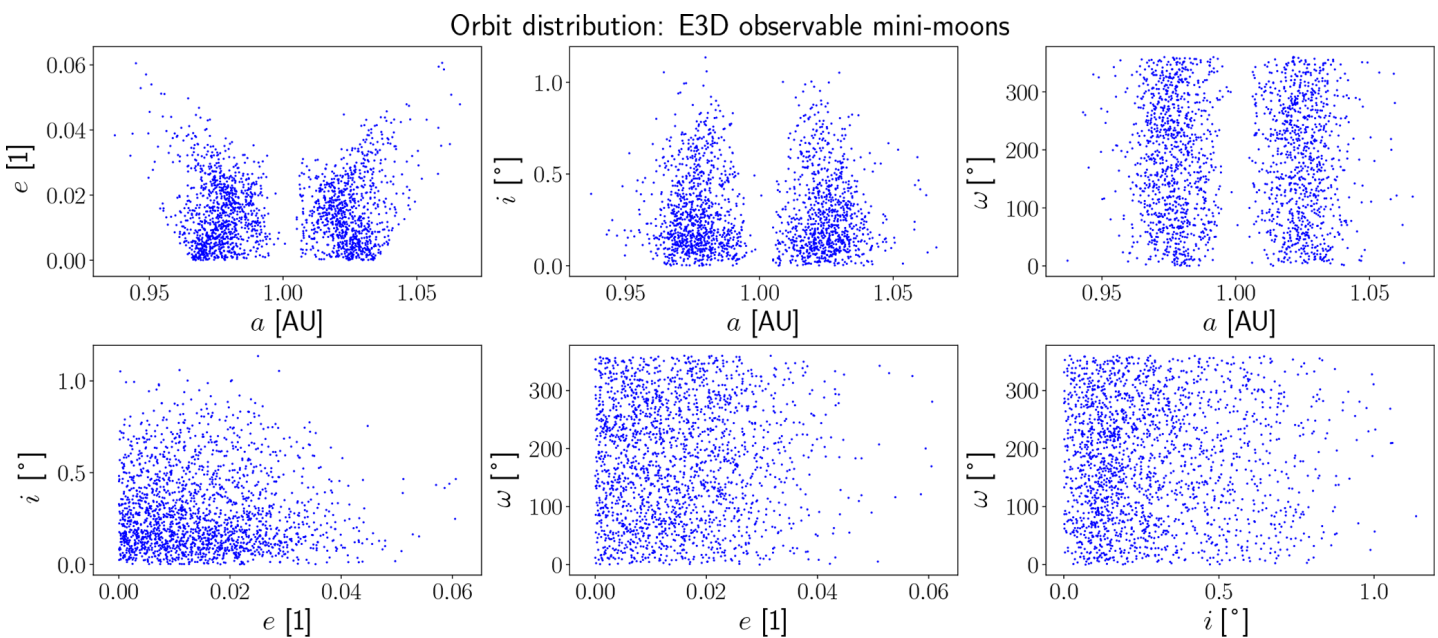

Figure 7. Orbital element distribution of mini-moons that can be tracked by E3D. This is not the detected orbital element distribution but rather what part of the initial distribution is observable. This illustration should be compared to the initial distribution in Fig. 2.

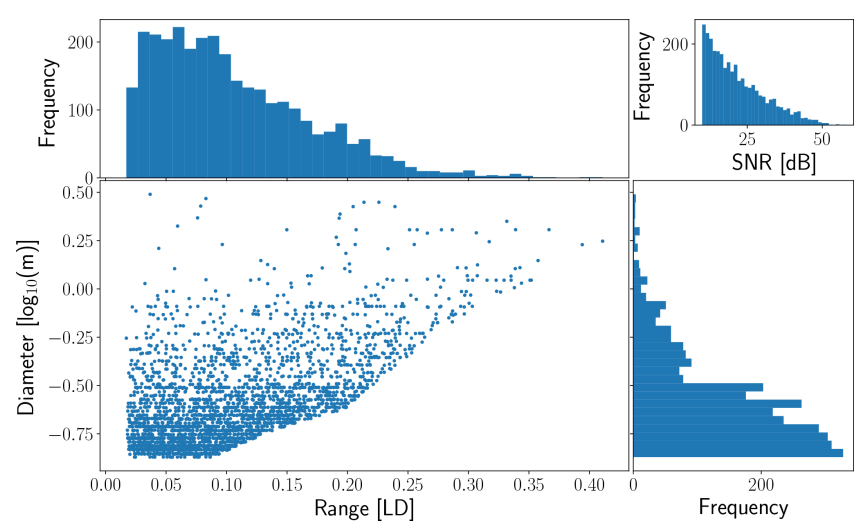

Figure 8. Distribution of ranges and sizes of possible observation windows. Also included is the distribution of peak SNR for these observation windows.

The distribution of ranges, sizes, and SNRs of all possible discovery windows is illustrated in Fig. 12.

As discussed in Sect. 4, a full sky scan by E3D could take approximately $5 \mathrm{~min}$. As the typical mini-moon observation window is on the order of hours, almost all of the possible serendipitous discoveries will be made if the radar performs a full sky scan every hour or half hour. This would use approximately $8 \%-16 \%$ of the available radar time.

Conducting routine NEO follow-up observations using E3D would allow for refined orbits and radar measurements of hundreds of mini-moons every year. Assuming a rotation rate of 1000 revolutions per day and using the observation window length for each possible tracking window, as illustrated in Fig. 11, we can estimate an average of $502 \mathrm{~h} \mathrm{yr}^{-1}$ would be spent on these observations. This is approximately $5.7 \%$ of the total radar time.

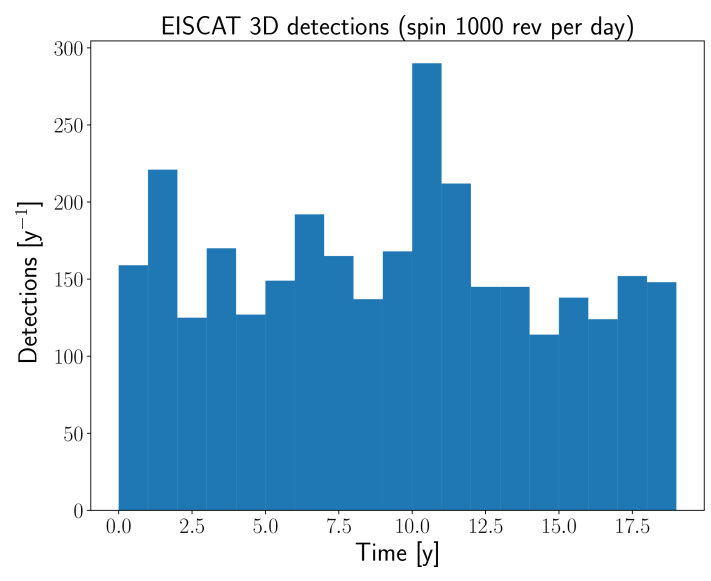

Figure 9. Yearly count of possible observation windows of minimoons, assuming a rotation rate of 1000 revolutions per day and that a prior orbit is available.

\section{Discussion}

For convenience, we have summarised the statistics from all three methods that were used to determine the observability of NEOs with E3D in Table 3. The advantage of using several different methods is not only that one can compare results but also that they inherently peer into different parts of the NEO population.

The fireball observations described in Sect. 2.1 is a sample from the subset of NEOs that make close approaches to Earth. In Brown et al. (2002) arguments are made that the measured population is unbiased. The known NEOs with close approaches described in Sect. 2.2 are also sampling the same subpopulation but in a different size range. This sample is biased and reduced due to limited observational capabilities. Only a fraction of all NEOs with close approaches are 


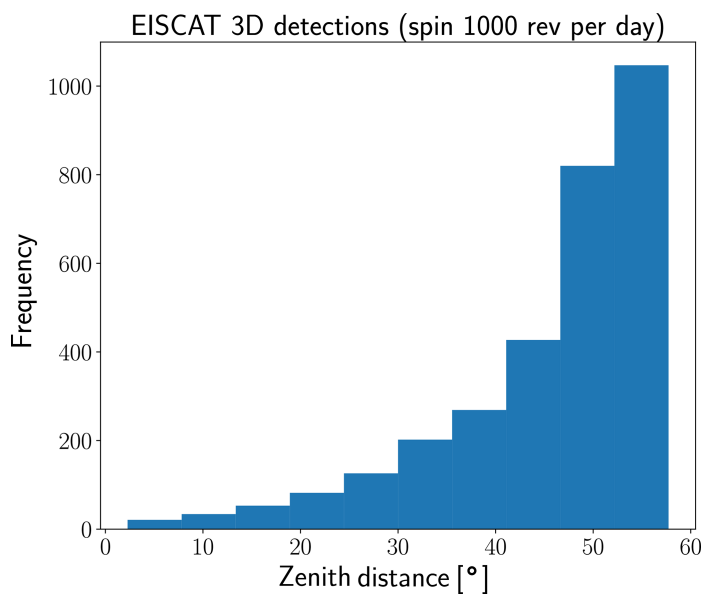

Figure 10. The zenith angle distribution of all possible observation windows, assuming a rotation rate of 1000 revolutions per day and that a prior orbit is available.

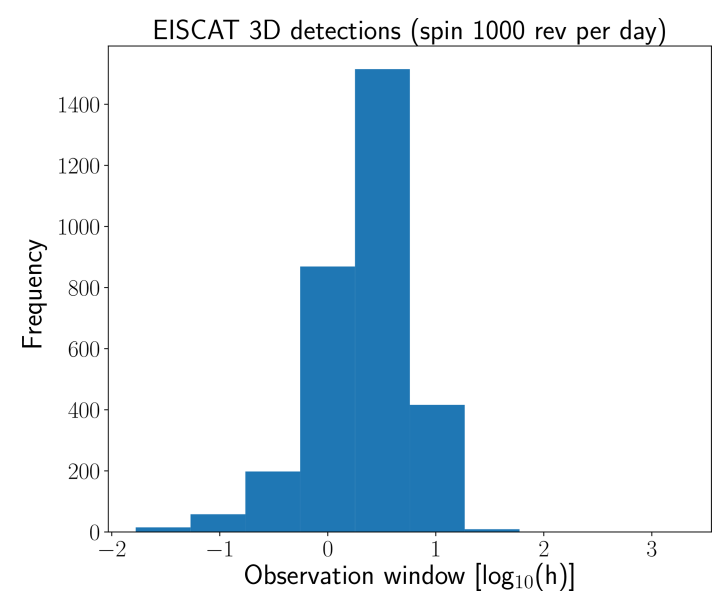

Figure 11. The length of each possible observation window that would provide a SNR above $10 \mathrm{~dB}$ assuming a rotation rate of 1000 revolutions per day and that a prior orbit is available.

currently detected. If the number of objects as a function of size scale is according to Eq. (1), the size ranges listed in Table 3 roughly translate to 2 orders of magnitude of fewer known NEOs than fireballs. As such, the 60-1200 discoveries from fireball statistics, versus 1 from the known NEOs with close approaches, are mutually consistent.

Based on the CNEOS catalogue, we have found that E3D can be used to observe $\approx 15$ objects per year. This number should be compared to $\approx 100$ by the Arecibo observatory. Another significant contribution is that smaller-sized objects are discoverable by the E3D system while conducting scans over its field of view, which opens the potential for a number of discoveries on the order of 1000 for objects in the $0.01-$ $1 \mathrm{~m}$ size range.

The difference in examined populations between the methods suggests that if routine NEO observations are imple-
Table 2. Summary counts of the mini-moon observability simulations spanning 19 years and propagated for 10 years. The number of observable objects is representative of the expected total number of real mini-moons that can be tracked by E3D in future if prior orbital elements are known. Each object can have more than one possible observation window and, on average, each object has approximately 1.5 tracking opportunities. The number of discoverable objects indicates how many serendipitous mini-moon detections can be made if an E3D scan hits the object with an integration time of $0.2 \mathrm{~s}$. Only a few objects have multiple discovery opportunities.

\begin{tabular}{lrr}
\hline $\begin{array}{l}\text { Observable objects } \\
\text { (out of 20 265) }\end{array}$ & $\begin{array}{r}\text { Observation } \\
\text { windows }\end{array}$ & $\begin{array}{r}\text { Rotation rate } \\
\left(\mathrm{rev} \mathrm{d}^{-1}\right)\end{array}$ \\
\hline 1999 & 3081 & 1000 \\
1594 & 2394 & 5000 \\
1461 & 2163 & 10000 \\
1058 & 1529 & 86400 \\
\hline Discoverable & Observation & $\begin{array}{r}\text { Coherent } \\
\text { objects }\end{array}$ \\
\hline 116 & 128 & 0.2 \\
\hline
\end{tabular}

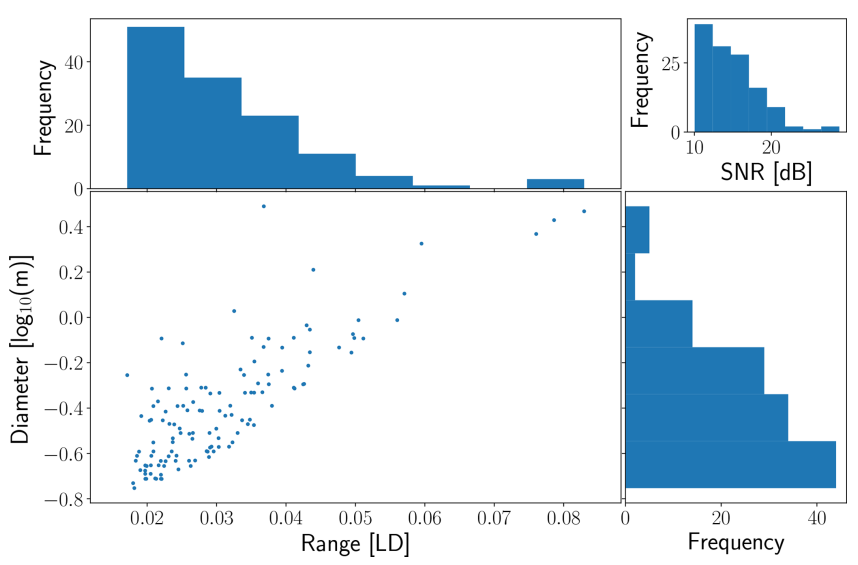

Figure 12. Distribution of ranges and sizes of possible discovery windows. Also included is the distribution of peak SNR for these discoveries.

mented at E3D, the simulation described in Sect. 7 should be recomputed using a representative subset of the debiased NEO population, such as the one presented in Granvik et al. (2018), extended to smaller sizes. This would provide guidance for the observation strategy and implementation of analysis and provide observation debiasing for E3D measurements. However, this would be more costly in terms of computational requirements, as the sampled population would need to be at least 2-3 orders of magnitude larger than the mini-moon population. The implementation of the mini-moon observability simulation using SORTS is fairly general. This means that in future, the same study can easily be performed for other radar systems as well. 
Table 3. Summary statistics from all three methods used to determine the observability of NEOs with E3D.

\begin{tabular}{|c|c|c|c|c|c|c|c|}
\hline \multirow{2}{*}{$\begin{array}{l}\text { Method } \\
\text { Rotation model }\end{array}$} & \multirow{2}{*}{$\begin{array}{l}\text { Fireball statistics } \\
\text { None }\end{array}$} & \multicolumn{2}{|c|}{ NEO close-encounter database } & \multicolumn{4}{|c|}{ Mini-moon simulations } \\
\hline & & $T_{\mathrm{r}}=0.005 \frac{D}{m} h$ & $T_{\mathrm{r}}=0.0001 \frac{D}{m} h$ & $1000 \frac{\mathrm{rev}}{d}$ & $5000 \frac{\mathrm{rev}}{d}$ & $10000 \frac{\mathrm{rev}}{d}$ & $86400 \frac{\mathrm{rev}}{d}$ \\
\hline Input population size & Density function & \multicolumn{2}{|c|}{1215} & \multicolumn{4}{|c|}{20272} \\
\hline Size range & $0.02-100 \mathrm{~m}$ & \multicolumn{2}{|c|}{$1.4-715 \mathrm{~m}$} & \multicolumn{4}{|c|}{$13 \mathrm{~cm}-4.3 \mathrm{~m}$} \\
\hline Detectable objects & - & 29 & 13 & 1999 & 1594 & 1461 & 1058 \\
\hline Detection rate $\frac{1}{v}$ & - & 29 & 13 & 162 & 126 & 114 & 80 \\
\hline Detection E3D usage & - & $0.3 \%-8 \%$ & - & $5.7 \%$ & $4.9 \%$ & $4.5 \%$ & $3.1 \%$ \\
\hline Discoverable objects & - & \multicolumn{2}{|l|}{ 年 } & \multicolumn{4}{|c|}{116} \\
\hline Discovery rate $\frac{1}{v}$ & $60-1200$ & \multicolumn{2}{|c|}{1} & \multicolumn{4}{|c|}{6.7} \\
\hline Discovery E3D usage & $100 \%$ & \multicolumn{2}{|c|}{$8 \%-16 \%$} & \multicolumn{4}{|c|}{$8 \%-16 \%$} \\
\hline
\end{tabular}

It was shown in Kastinen et al. (2019) that E3D is expected to regularly detect hard target echoes from space debris and other artificial objects in the Earth's orbit. NEOs and mini-moons need to be robustly separated from these artificial objects for discovery operations to be successful. Space debris is mainly confined to two regions, namely close to Earth $\left(<3 \times 10^{3} \mathrm{~km}\right.$ altitude) or close to geostationary orbit ( $\sim 3.6 \times 10^{4} \mathrm{~km}$ altitude; Krisko, 2010; Flegel et al., 2009). Our results show that the typical mini-moon or NEO detection will be made at altitudes larger than these regions and up to $3.8 \times 10^{5} \mathrm{~km}$ altitude. Thus, the range to the target can be used as an initial NEO and mini-moon identification. If the orbit of the object can be determined, this would be a very reliable method of identification as NEOs and mini-moons generally have vastly different orbits compared to space debris (Fedorets et al., 2017).

Our results indicate that $\mathrm{E} 3 \mathrm{D}$ can provide valuable and unique follow-up measurements of mini-moons and NEOs with close approaches. It also shows that, even though discovering mini-moons is sparse, discovery and scanning for the combined population of mini-moons and generic NEOs may be very cost effective as this is inherently dual usage with space debris observations. That is, the same radar pulses and survey patterns can be used for discovering objects from all of the above-mentioned populations. Even the discovery of a single new mini-moon would be significant since, to date, only two have been discovered.

The scientific gain from tracking operations at E3D can be summarised as being efficient, high-quality orbit determination and, if the target is sufficiently larger than the wavelength, novel data on surface properties and rotation rates. There are currently not many methods that can discover smaller NEOs unless they collide with the Earth's atmosphere, as shown by the low number of discovered minimoons. As such, the scientific gain from discovery operations is in essence the discovery itself, i.e. the observation capability of a population otherwise not observable. If the objects are larger, they can be detected with higher probability using optical methods. In these cases, radar observations are still valuable for the same reasons as tracking operations are.
The feasibility of a follow-up observation programme can be tested in practice by using known space debris objects with large distances. For example, large objects with Molniya orbits are good candidates for testing the detection capabilities of faraway objects over long integration times.

It is also valuable to note that E3D will observe over 1000 meteors per hour (Pellinen-Wannberg et al., 2016). Radar meteors are a direct measurement of the NEO population that makes close approaches to Earth but at much smaller sizes than the ones examined here. In Fedorets et al. (2017) $1.46 \%$ of all temporarily captured fly-bys and $0.61 \%$ of all mini-moons impacted Earth. Due to the tri-static capability of E3D, the inferred orbital elements will be of very high quality, and it may be possible to trace meteors back to the mini-moon population.

\section{Conclusions}

Our results indicate that it is plausible that E3D can be used to discover NEOs with diameters $D>0.01 \mathrm{~m}$. All of the populations studied predicted that E3D would discover NEOs by using an all-sky radar survey. A rough estimate of up to 1200 detections per year is possible when using $100 \%$ of the radar time at full-duty cycle. This estimate is based on a very simplistic model, which neglects many important details. However, these results are encouraging and suggest that the radar detectability of NEOs should be investigated further. The capability of discovering NEOs would have several advantages. Radars can observe in the day-lit hemisphere. The objects that can be found using radar are mostly smaller than the ones detectable using telescopic surveys, and observations of accurate orbital elements of objects in this size range are very scarce.

The study of the mini-moon subset of the NEO population indicates that a significant fraction of objects could be tracked, with 80-160 observing opportunities per year, assuming that the objects have been previously discovered. There is currently only one mini-moon in the Earth's orbit, but it is no longer observable using EISCAT UHF or E3D 
due to the long range when the object is in the radar field of view. However, there will be more opportunities in future for such observations as new mini-moons are discovered $(\mathrm{Fe}$ dorets et al., 2020). Our study shows that an E3D-based radar search for mini-moons is one potential way for discovering these objects. Our simulation suggests that there are approximately seven discoveries per year with a $8 \%-16 \%$ utilisation of radar resources. In addition to utilising dedicated observing modes for these searches, it should be feasible to also perform a secondary analysis to search for NEOs when running the radar in ionospheric mode.

Our study shows that establishing a post-discovery NEO tracking programme that uses close-approach predictions is feasible. Such an initiative could already be commenced with the existing EISCAT UHF radar, which is only slightly less sensitive than the upcoming E3D radar for this purpose. We estimate that roughly $0.5 \%$ to $1 \%$ of the 2000 objects discovered annually could be tracked using the EISCAT UHF or E3D radars, based on close approaches in 2019. The need for radar resources is minimal, with only a few $4-8 \mathrm{~h}$ observing windows each month. However, the observations would need to be scheduled on short notice, using an automated alert system that notifies of upcoming observing possibilities (cf. Solin and Granvik, 2018). The measurements would yield accurate orbital elements of NEOs but possibly also estimates for sizes and rotation rates. The dual polarisation capability of E3D could also be used to study the composition of these objects.

Data availability. The underlying research data came from two sources, namely the CNEOS database and a mini-moon model provided by Mikael Granvik, which is not publicly available. The CNEOS database is available online at https://cneos.jpl.nasa.gov/ ca/ (NASA JPL, 2020).

Author contributions. DK performed the numerical simulations of mini-moon observations by E3D in Sect. 7. TT performed the detectability calculation for objects within the CNEOS catalogue in Sect. 6. JV provided the SNR calculations in Sect. 3 and estimated the number of NEOs serendipitously detectable in Sect. 5. MG provided the mini-moon population model described in Sect. 2.3. All the authors contributed to the preparation of the paper and interpretation of the results.

Competing interests. Juha Vierinen is on the editorial board of the journal.

Special issue statement. This article is part of the "Special Issue on the joint 19th International EISCAT Symposium and 46th Annual European Meeting on Atmospheric Studies by Optical Methods". It is a result of the 19th International EISCAT Symposium 2019 and 46th Annual European Meeting on Atmospheric Studies by Optical Methods, Oulu, Finland, 19-23 August 2019.

Review statement. This paper was edited by Petr Pisoft and reviewed by Peter Brown and one anonymous referee.

\section{References}

Balanis, C. A.: Advanced engineering electromagnetics, John Wiley \& Sons, Hoboken, New Jersey, 1999.

Banka, D., Leushacke, L., and Mehrholz, D.: Beam-parkexperiment-1/2000 with TIRA, Space Debris, 2, 83-96, 2000.

Beech, M. and Brown, P.: Fireball flickering: the case for indirect measurement of meteoroid rotation rates, Planet. Space Sci., 48, 925-932, https://doi.org/10.1016/S0032-0633(00)00058-1, 2000.

Benner, L. A. M., Busch, M. W., Giorgini, J. D., Taylor, P. A., and Margot, J. L.: Radar Observations of Near-Earth and MainBelt Asteroids, in: Asteroids IV, edited by: Michel, P., DeMeo, F. E., and Bottke, W. F., University of Arizona Press, Tucson, 165-182, https://doi.org/10.2458/azu_uapress_9780816532131ch009, 2015.

Bolin, B., Jedicke, R., Granvik, M., Brown, P., Howell, E., Nolan, M. C., Jenniskens, P., Chyba, M., Patterson, G., and Wainscoat, R.: Detecting Earth's temporarilycaptured natural satellites-Minimoons, Icarus, 241, 280-297, https://doi.org/10.1016/j.icarus.2014.05.026, 2014.

Bottke, W. F., Morbidelli, A., Jedicke, R., Petit, J.-M., Levison, H. F., Michel, P., and Metcalfe, T. S.: Debiased Orbital and Absolute Magnitude Distribution of the Near-Earth Objects, Icarus, 156, 399-433, https://doi.org/10.1006/icar.2001.6788, 2002.

Braun, G.: GESTRA - Experimental space monitoring radar, available at: https://event.dlr.de/en/ila2018/gestra/ (last access: 13 March 2020), 2018.

Brown, P., Spalding, R., ReVelle, D. O., Tagliaferri, E., and Worden, S.: The flux of small near-Earth objects colliding with the Earth, Nature, 420, 294-296, https://doi.org/10.1038/nature01238, 2002.

Busch, M. W., Kulkarni, S. R., Brisken, W., Ostro, S. J., Benner, L. A., Giorgini, J. D., and Nolan, M. C.: Determining asteroid spin states using radar speckles, Icarus, 209, 535-541, 2010.

Campbell, B. A.: Planetary geology with imaging radar: insights from earth-based lunar studies, 2001-2015, Astr. Soc. P., 128, 062001, https://doi.org/10.1088/1538-3873/128/964/062001, 2016.

Čapek, D.: Rotation of cometary meteoroids, Astron. Astrophys., 568, A39, https://doi.org/10.1051/0004-6361/201423857, 2014.

Chesley, S. R. and Chodas, P. W.: Asteroid close approaches: analysis and potential impact detection, in: Asteroids III, University of Arizona Press, Tucson, AZ, USA, 55-69, 2002.

Fedorets, G., Granvik, M., and Jedicke, R.: Orbit and size distributions for asteroids temporarily captured by the Earth-Moon system, Icarus, 285, 83-94, https://doi.org/10.1016/j.icarus.2016.12.022, 2017.

Fedorets, G., Granvik, M., Jones, R. L., Jurić, M., and Jedicke, R.: Discovering Earth's transient moons with the 
Large Synoptic Survey Telescope, Icarus, 338, 113517, https://doi.org/10.1016/j.icarus.2019.113517, 2020.

Flegel, S., Gelhaus, J., Wiedemann, C., Vorsmann, P., Oswald, M., Stabroth, S., Klinkrad, H., and Krag, H.: Invited Paper: The MASTER-2009 Space Debris Environment Model, in: Fifth European Conference on Space Debris, ESOC, Darmstadt, Germany, Vol. 672 of ESA Special Publication, p. 15, 2009.

Fowler, J. and Chillemi, J.: IRAS asteroid data processing, edited by: Tedesco, E. F., Veeder, G. J., Fowler, J. W., and Chillemi, J. R., The IRAS Minor Planet Survey, Technical Report PL-TR-922049, Phillips Laboratory, Hanscom AF Base, MA, 1992.

Granvik, M., Vaubaillon, J., and Jedicke, R.: The population of natural Earth satellites, Icarus, 218, 262-277, https://doi.org/10.1016/j.icarus.2011.12.003, 2012.

Granvik, M., Jedicke, R., Bolin, B., Chyba, M., and Patterson, G.: Earth's Temporarily-Captured Natural Satellites - The First Step towards Utilization of Asteroid Resources, Asteroids: Prospective Energy and Material Resources, edited by: Badescu, V., Springer, Berlin, 151-167, https://doi.org/10.1007/978-3-64239244-3_6, 2013.

Granvik, M., Morbidelli, A., Jedicke, R., Bolin, B., Bottke, W. F., Beshore, E., Vokrouhlický, D., Delbò, M., and Michel, P.: Supercatastrophic disruption of asteroids at small perihelion distances, Nature, 530, 303-306, https://doi.org/10.1038/nature16934, 2016.

Granvik, M., Morbidelli, A., Jedicke, R., Bolin, B., Bottke, W. F., Beshore, E., Vokrouhlický, D., Nesvorný, D., and Michel, P.: Debiased orbit and absolute-magnitude distributions for near-Earth objects, Icarus, 312, 181-207, https://doi.org/10.1016/j.icarus.2018.04.018, 2018.

Harris, A. W. and Harris, A. W.: On the Revision of Radiometric Albedos and Diameters of Asteroids, Icarus, 126, 450-454, https://doi.org/10.1006/icar.1996.5664, 1997.

Ivezić, Ž., Kahn, S. M., Tyson, J. A., Abel, B., Acosta, E., Allsman, R., Alonso, D., AlSayyad, Y., Anderson, S. F., Andrew, J., Angel, J. R. P., Angeli, G. Z., Ansari, R., Antilogus, P., Araujo, C., Armstrong, R., Arndt, K. T., Astier, P., Aubourg, É., Auza, N., Axelrod, T. S., Bard, D. J., Barr, J. D., Barrau, A., Bartlett, J. G., Bauer, A. E., Bauman, B. J., Baumont, S., Bechtol, E., Bechtol, K., Becker, A. C., Becla, J., Beldica, C., Bellavia, S., Bianco, F. B., Biswas, R., Blanc, G., Blazek, J., Bland ford, R. D., Bloom, J. S., Bogart, J., Bond, T. W., Booth, M. T., Borgland, A. W., Borne, K., Bosch, J. F., Boutigny, D., Brackett, C. A., Bradshaw, A., Brand t, W. N., Brown, M. E., Bullock, J. S., Burchat, P., Burke, D. L., Cagnoli, G., Calabrese, D., Callahan, S., Callen, A. L., Carlin, J. L., Carlson, E. L., Chand rasekharan, S., CharlesEmerson, G., Chesley, S., Cheu, E. C., Chiang, H.-F., Chiang, J., Chirino, C., Chow, D., Ciardi, D. R., Claver, C. F., CohenTanugi, J., Cockrum, J. J., Coles, R., Connolly, A. J., Cook, K. H., Cooray, A., Covey, K. R., Cribbs, C., Cui, W., Cutri, R., Daly, P. N., Daniel, S. F., Daruich, F., Daubard, G., Daues, G., Dawson, W., Delgado, F., Dellapenna, A., de Peyster, R., de Val-Borro, M., Digel, S. W., Doherty, P., Dubois, R., Dubois-Felsmann, G. P., Durech, J., Economou, F., Eifler, T., Eracleous, M., Emmons, B. L., Fausti Neto, A., Ferguson, H., Figueroa, E., Fisher-Levine, M., Focke, W., Foss, M. D., Frank, J., Freemon, M. D., Gangler, E., Gawiser, E., Geary, J. C., Gee, P., Geha, M., Gessner, C. J. B., Gibson, R. R., Gilmore, D. K., Glanzman, T., Glick, W., Goldina, T., Goldstein, D. A., Goodenow, I., Graham, M. L., Gressler,
W. J., Gris, P., Guy, L. P., Guyonnet, A., Haller, G., Harris, R., Hascall, P. A., Haupt, J., Hernand ez, F., Herrmann, S., Hileman, E., Hoblitt, J., Hodgson, J. A., Hogan, C., Howard, J. D., Huang, D., Huffer, M. E., Ingraham, P., Innes, W. R., Jacoby, S. H., Jain, B., Jammes, F., Jee, M. J., Jenness, T., Jernigan, G., Jevremović, D., Johns, K., Johnson, A. S., Johnson, M. W. G., Jones, R. L., Juramy-Gilles, C., Jurić, M., Kalirai, J. S., Kallivayalil, N. J., Kalmbach, B., Kantor, J. P., Karst, P., Kasliwal, M. M., Kelly, H., Kessler, R., Kinnison, V., Kirkby, D., Knox, L., Kotov, I. V., Krabbendam, V. L., Krughoff, K. S., Kubánek, P., Kuczewski, J., Kulkarni, S., Ku, J., Kurita, N. R., Lage, C. S., Lambert, R., Lange, T., Langton, J. B., Le Guillou, L., Levine, D., Liang, M., Lim, K.-T., Lintott, C. J., Long, K. E., Lopez, M., Lotz, P. J., Lupton, R. H., Lust, N. B., MacArthur, L. A., Mahabal, A., Mand elbaum, R., Markiewicz, T. W., Marsh, D. S., Marshall, P. J., Marshall, S., May, M., McKercher, R., McQueen, M., Meyers, J., Migliore, M., Miller, M., Mills, D. J., Miraval, C., Moeyens, J., Moolekamp, F. E., Monet, D. G., Moniez, M., Monkewitz, S., Montgomery, C., Morrison, C. B., Mueller, F., Muller, G. P., Muñoz Arancibia, F., Neill, D. R., Newbry, S. P., Nief, J.-Y., Nomerotski, A., Nordby, M., O'Connor, P., Oliver, J., Olivier, S. S., Olsen, K., O’Mullane, W., Ortiz, S., Osier, S., Owen, R. E., Pain, R., Palecek, P. E., Parejko, J. K., Parsons, J. B., Pease, N. M., Peterson, J. M., Peterson, J. R., Petravick, D. L., Libby Petrick, M. E., Petry, C. E., Pierfederici, F., Pietrowicz, S., Pike, R., Pinto, P. A., Plante, R., Plate, S., Plutchak, J. P., Price, P. A., Prouza, M., Radeka, V., Rajagopal, J., Rasmussen, A. P., Regnault, N., Reil, K. A., Reiss, D. J., Reuter, M. A., Ridgway, S. T., Riot, V. J., Ritz, S., Robinson, S., Roby, W., Roodman, A., Rosing, W., Roucelle, C., Rumore, M. R., Russo, S., Saha, A., Sassolas, B., Schalk, T. L., Schellart, P., Schindler, R. H., Schmidt, S., Schneider, D. P., Schneider, M. D., Schoening, W., Schumacher, G., Schwamb, M. E., Sebag, J., Selvy, B., Sembroski, G. H., Seppala, L. G., Serio, A., Serrano, E., Shaw, R. A., Shipsey, I., Sick, J., Silvestri, N., Slater, C. T., Smith, J. A., Smith, R. C., Sobhani, S., Soldahl, C., Storrie-Lombardi, L., Stover, E., Strauss, M. A., Street, R. A., Stubbs, C. W., Sullivan, I. S., Sweeney, D., Swinbank, J. D., Szalay, A., Takacs, P., Tether, S. A., Thaler, J. J., Thayer, J. G., Thomas, S., Thornton, A. J., Thukral, V., Tice, J., Trilling, D. E., Turri, M., Van Berg, R., Vanden Berk, D., Vetter, K., Virieux, F., Vucina, T., Wahl, W., Walkowicz, L., Walsh, B., Walter, C. W., Wang, D. L., Wang, S.-Y., Warner, M., Wiecha, O., Willman, B., Winters, S. E., Wittman, D., Wolff, S. C., WoodVasey, W. M., Wu, X., Xin, B., Yoachim, P., and Zhan, H.: LSST: From Science Drivers to Reference Design and Anticipated Data Products, Astrophys. J., 873, 111, https://doi.org/10.3847/15384357/ab042c, 2019.

Jedicke, R., Bolin, B. T., Bottke, W. F., Chyba, M., Fedorets, G., Granvik, M., Jones, L., and Urrutxua, H.: Earth's Minimoons: Opportunities for Science and Technology, Frontiers in Astronomy and Space Sciences, 5, 13, https://doi.org/10.3389/fspas.2018.00013, 2018.

Kaasalainen, M. and Viikinkoski, M.: Shape reconstruction of irregular bodies with multiple complementary data sources, Astron. Astrophys., 543, A97, https://doi.org/10.1051/00046361/201219267, 2012.

Kastinen, D., Vierinen, J., Kero, J., Hesselbach, S., Grydeland, T., and Krag, H.: Next-generation Space Object Radar Tracking 
Simulator: SORTS++, European Space Agency, ESOC, Darmstadt, Germany, 2019.

Kessler, D. J., Landry, P. M., Gabbard, J. R., and Moran, J. L. T.: Ground radar detection of meteoroids in space, in: Solid Particles in the Solar System, edited by: Halliday, I. and McIntosh, B. A., Vol. 90 of IAU Symposium, 137-139, Proceedings of the Symposium, Ottawa, Canada, D. Reidel Publishing Co., Dordrecht, 1980.

Krisko, P. H.: NASA's New Orbital Debris Engineering Model, ORDEM 2010, in: Making Safety Matter, Proceedings of the fourth IAASS Conference, 19-21 May 2010, Huntsville, AL, edited by: Lacoste-Francis, H., ESA-SP Vol. 680, p. 50, 2010.

Krisko, P. H.: The new NASA orbital debris engineering model ORDEM 3.0, in: AIAA/AAS Astrodynamics Specialist Conference, San Diego, CA, p. 4227, American Institute of Aeronautics and Astronautics, Reston (HQ), VA, United States, https://doi.org/10.2514/6.2014-4227, 2014.

Kwiatkowski, T., Kryszczyńska, A., Polińska, M., Buckley, D. A. H., O'Donoghue, D., Charles, P. A., Crause, L., Crawford, S., Hashimoto, Y., Kniazev, A., Loaring, N., Romero Colmenero, E., Sefako, R., Still, M., and Vaisanen, P.: Photometry of 2006 $\mathrm{RH}\{120\}$ : an asteroid temporary captured into a geocentric orbit, Astron. Astrophys., 495, 967-974, https://doi.org/10.1051/00046361:200810965, 2009.

Li, A., Close, S., and Markannen, J.: EISCAT Space Debris after the International Polar Year (IPY), in: Conference Proceedings from IAC, Naples, Italy, Vol. 12, p. A6, International Astronautical Federation, Paris, France, 2012.

Liou, J.-C., Matney, M. J., Anz-Meador, P. D., Kessler, D., Jansen, M., and Theall, J. R.: The new NASA orbital debris engineering model ORDEM2000, 2002.

Markkanen, J., Jehn, R., and Krag, H.: EISCAT space debris during the IPY - a 5000 hour campaign, in: Proceedings of the Fifth European Conference on Space Debris, 30 March-2 April 2009, Darmstadt, Germany, edited by: Lacoste, H., ESA-SP Vol. 672, European Space Agency, 2009.

McCrea, I., Aikio, A., Alfonsi, L., Belova, E., Buchert, S., Clilverd, M., Engler, N., Gustavsson, B., Heinselman, C., Kero, J., Kosch, M., Lamy, H., Leyser, T., Ogawa, Y., Oksavik, K., PellinenWannberg, A., Pitout, F., Rapp, M., Stanislawska I., and Vierinen, J.: The science case for the EISCAT_3D radar, Prog. Earth Planet. Sci., 2, 21, https://doi.org/10.1186/s40645-015-0051-8, 2015.

Morbidelli, A., Delbo, M., Granvik, M., Bottke, W. F., Jedicke, R., Bolin, B., Michel, P., and Vokrouhlicky, D.: Debiased albedo distribution for Near Earth Objects, Icarus, 340, 113631, https://doi.org/10.1016/j.icarus.2020.113631, 2020.

Naidu, S. P., Benner, L. A., Margot, J.-L., Busch, M. W., and Taylor, P. A.: Capabilities of Earth-based radar facilities for near-Earth asteroid observations, Astron. J., 152, 4, https://doi.org/10.3847/0004-6256/152/4/99, 2016.

NASA JPL: CNEOS NEO Earth Close Approaches, available at: https://cneos.jpl.nasa.gov/ca/, last access: 13 March 2020.

Ostro, S. J.: Planetary radar, NASA Jet Propulsion Laboratory, Technical Reports Server, Pasadena, California, United States, available at: https://trs.jpl.nasa.gov/ (last access: 10 July 2020), 1992.
Ostro, S. J.: The role of groundbased radar in near-Earth object hazard identification and mitigation, Univ. of Arizona Press, Pasadena, CA, United States, 1994.

Ostro, S. J., Connelly, R., and Belkora, L.: Asteroid shapes from radar echo spectra: A new theoretical approach, Icarus, 73, 1524, 1988.

Pellinen-Wannberg, A., Kero, J., Häggström, I., Mann, I., and Tjulin, A.: The forthcoming EISCAT_3D as an extra-terrestrial matter monitor, Planet. Space Sci., 123, 33-40, 2016.

Pravec, P., Harris, A. W., and Michalowski, T.: Asteroid rotations, in: Asteroids III, edited by: Bottke Jr., W. F., Cellino, A., Paolicchi, P., and Binzel, R. P., University of Arizona Press, Tucson, 113-122, 2002.

Rein, H. and Liu, S.-F.: REBOUND: an open-source multi-purpose N-body code for collisional dynamics, Astron. Astrophys., 537, A128, https://doi.org/10.1051/0004-6361/201118085, 2012.

Rein, H. and Spiegel, D. S.: IAS15: a fast, adaptive, high-order integrator for gravitational dynamics, accurate to machine precision over a billion orbits, Mon. Not. R. Astron. Soc., 446, 1424-1437, https://doi.org/10.1093/mnras/stu2164, 2015.

Solin, O. and Granvik, M.: Monitoring near-Earth-object discoveries for imminent impactors, Astron. Astrophys., 616, A176, https://doi.org/10.1051/0004-6361/201832747, 2018.

Taylor, P., Rivera-Valentín, E. G., Bonsall, A., Becker, T. M., Benner, A., Bhiravarasu, S. S., Brozovic, M., Busch, M. W., Giorgini, J. D., Harris, A. W., Magri, C., Mainzer, A. K., Margot, J.-L., Marshall, S. E., Masiero, J. R., Naidu, S. P., Nolan, M. C., Patterson, G. W., Prockter, L. M., Sizemore, H. G., Swindle, T. D., Venditti, F. C. F., and Virkki, A. K.: Planetary Radar Astronomy with Ground-Based Astrophysical Assets, Astro2020 Science White Paper, American Astronomical Society, Washington, D.C., USA, 2019.

Taylor, P. A., Howell, E. S., Nolan, M. C., and Thane, A. A.: The Shape and Spin Distributions of Near-Earth Asteroids Observed with the Arecibo Radar System, in: American Astronomical Society Meeting Abstracts \#220, Vol. 220 of American Astronomical Society Meeting Abstracts, p. 128.02, American Astronomical Society, Washington, D.C., USA, 2012.

Thompson, T. W., Campbell, B. A., and Bussey, D. B. J.: 50 Years of Arecibo Lunar radar mapping, URSI Radio Science Bulletin, 2016, 23-35, 2016.

Vierinen, J.: Indian anti-satellite debris measured with the EISCAT Troms $\emptyset$ radar, available at: http://www.radio-science.net/ 2019/04/indian-anti-satellite-debris-measured.html (last access: 13 March 2020), 2019.

Vierinen, J. and Lehtinen, M. S.: 32-cm wavelength radar mapping of the moon, in: 2009 European Radar Conference (EuRAD), Rome, Italy, 222-225, IEEE, 2009.

Vierinen, J., Markkanen, J., and Krag, H.: High power large aperture radar observations of the Iridium-COSMOS collision, in: Proceedings of the Fifth European Conference on Space Debris, 30 March-2 April 2009, Darmstadt, Germany, edited by: Lacoste, H., ESA-SP Vol. 672, European Space Agency, 2009.

Vierinen, J., Markkanen, J., Krag, H., Siminski, J., and Mancas, A.: Use of EISCAT 3D for Observations of Space Debris, 7th European Conference on Space Debris ESA/ESOC, Darmstadt/Germany 18-21 April 2017, ESA Space Debris Office, 2017 a. 
Vierinen, J., Tveito, T., Gustavsson, B., Kesaraju, S., and Milla, M.: Radar images of the Moon at 6-meter wavelength, Icarus, 297, 179-188, 2017b.

Vierinen, J., Kastinen, D., Kero, J., Grydeland, T., McKay, D., Røynestad, E., Hesselbach, S., Kebschull, C., and Krag, H.: EISCAT 3D Performance Analysis, Tech. rep., ESA/ESOC Technical Management, Darmstadt, Germany, 2019a.

Vierinen, J., Kastinen, D., Markkanen, J., Grydeland, T., Kero, J., Horstmann, A., Hesselbach, S., Kebschull, C., Røynestad, E., and Krag, H.: 2018 Beam-park observations of space debris with the EISCAT radars, European Space Agency, ESOC, Darmstadt, Germany, 2019b.
Zellner, B. and Gradie, J.: Minor planets and related objects. XXPolarimetric evidence for the albedos and compositions of 94 asteroids, Astron. J., 81, 262-280, 1976. 